\title{
On the Fefferman-Phong Inequality and a Wiener-type Algebra of Pseudodifferential Operators
}

\author{
By
}

\author{
Nicolas LeRner* and Yoshinori Morimoto**
}

\begin{abstract}
We provide an extension of the Fefferman-Phong inequality to nonnegative symbols whose fourth derivative belongs to a Wiener-type algebra of pseudodifferential operators introduced by J. Sjöstrand. As a byproduct, we obtain that the number of derivatives needed to get the classical Fefferman-Phong inequality in $d$ dimensions is bounded above by $2 d+4+\epsilon$. Our method relies on some refinements of the Wick calculus, which is closely linked to Gabor wavelets. Also we use a decomposition of $C^{3,1}$ nonnegative functions as a sum of squares of $C^{1,1}$ functions with sharp estimates. In particular, we prove that a $C^{3,1}$ nonnegative function $a$ can be written as a finite sum $\sum b_{j}^{2}$, where each $b_{j}$ is $C^{1,1}$, but also where each function $b_{j}^{2}$ is $C^{3,1}$. A key point in our proof is to give some bounds on $\left(b_{j}^{\prime} b_{j}^{\prime \prime}\right)^{\prime}$ and on $\left(b_{j} b_{j}^{\prime \prime}\right)^{\prime \prime}$.
\end{abstract}

\section{Contents}

$\S 1$. Introduction and Statement of the Results

$\S 1.1$. The Fefferman-Phong inequality and Bony's result

$\S 1.2$. Sjöstrand algebra of pseudodifferential operators

$\S 1.3$. The main result

$\S 2$. The Wick Calculus of Pseudodifferential Operators

§2.1. Definitions

§2.2. Sharp estimates for the remainders

$\S 2.3$. On the composition formula for the Wick quantization

Communicated by T. Kawai. Received March 16, 2006.

2000 Mathematics Subject Classification(s): 47G30, 35S05, 42C15, 47B38, 81R30, 81S30.

* Projet analyse fonctionnelle, Institut de Mathématiques de Jussieu, Université Pierre et Marie Curie (Paris 6), 175 rue du Chavaleret - 75013 Paris, France.

e-mail: lerner@math.jussieu.fr http://www.math.jussieu.fr// lerner/

** Graduate School of Human and Environmental. Studies, Kyoto University, Kyoto 6068501, Japan.

e-mail: morimoto@math.h.kyoto-u.ac.jp

http://www.math.h.kyoto-u.ac.jp/ morimoto

(c) 2007 Research Institute for Mathematical Sciences, Kyoto University. All rights reserved. 
$\S 3 . \quad$ The Proof

§3.1. Nonnegative functions as sum of squares

§3.2. Application of the Wick calculus: proof of Theorem 1.3.1

$\S 3.3$. Proof of Corollary 1.3.2

A. Appendix

$\S$ A.1. On nonnegative functions

$\S$ A.2. More properties of the algebra $\mathcal{A}$

$\S$ A.3. On Leibniz formulæ

$\S$ A.4. Symmetric $k$-tensors as sum of $k$-th powers

$\S$ A.5. From discrete sums to finite sums

\section{$\S 1$. Introduction and Statement of the Results}

\section{§1.1. The Fefferman-Phong inequality and Bony's result}

Let us consider a classical second-order symbol $a(x, \xi)$, i.e. a smooth function defined on $\mathbb{R}^{n} \times \mathbb{R}^{n}$ such that, for all multi-indices $\alpha, \beta$

$$
\left|\left(\partial_{\xi}^{\alpha} \partial_{x}^{\beta} a\right)(x, \xi)\right| \leq C_{\alpha \beta}(1+|\xi|)^{2-|\alpha|} .
$$

The Fefferman-Phong inequality states that, if $a$ satisfies (1.1.1) and is a nonnegative function, there exists $C$ such that, for all $u \in \mathcal{S}\left(\mathbb{R}^{n}\right)$,

$$
\operatorname{Re}\langle a(x, D) u, u\rangle_{L^{2}\left(\mathbb{R}^{n}\right)}+C\|u\|_{L^{2}\left(\mathbb{R}^{n}\right)}^{2} \geq 0,
$$

or equivalently (with an a priori different constant $C$ )

$$
a^{w}+C \geq 0
$$

where $a^{w}$ stands for the Weyl quantization ${ }^{1}$ of $a$,

$$
\left(a^{w} u\right)(x)=\iint e^{2 i \pi(x-y) \xi} a\left(\frac{x+y}{2}, \xi\right) u(y) d y d \xi .
$$

The constant $C$ in (1.1.2-3) depends only a finite number of $C_{\alpha \beta}$ in (1.1.1). Let us ask our first question:

How many derivatives of a in (1.1.1) are needed to control $C$ in (1.1.2)?

\footnotetext{
${ }^{1}$ The standard quantization $a(x, D)$ reads $(a(x, D) u)(x)=\int e^{2 i \pi x \xi} a(x, \xi) \hat{u}(\xi) d \xi$.
} 
Looking at the proof by C. Fefferman and D. H. Phong [FP] (see also Theorem 18.6.8 in the third volume of [H2]), it seems clear that the number $N$ of derivatives of $a$ needed to control $C$ should be

$$
N=4+\nu(n), \quad \nu \text { depending on the dimension } n .
$$

Since the proof is using an induction on the dimension, it is not completely obvious to answer to our question with a reasonably simple $\nu$. On the other hand, J.-M. Bony proved in [Bo1] (Théorème 3.2 ) the following result: if $a(x, \xi)$ is a nonnegative smooth function defined on $\mathbb{R}^{n} \times \mathbb{R}^{n}$ such that

$$
\left|\left(\partial_{\xi}^{\alpha} \partial_{x}^{\beta} a\right)(x, \xi)\right| \leq C_{\alpha \beta}, \quad \text { for }|\alpha|+|\beta| \geq 4,
$$

then the conclusions (1.1.2-3) hold. This result shows an interesting twofold phenomenon:

- Only derivatives with order larger than 4 are needed.

- The control of these derivatives is quite weak, of type $S_{0,0}^{0}$. In particular, the derivatives of large order do not get small (the class $S_{0,0}^{0}$ does not have an asymptotic calculus).

Our answer to the question (1.1.4) is $4+2 n+\epsilon$ (for any positive $\epsilon$ ). However, we shall in fact prove a much more precise result involving a Wiener-type algebra introduced by J. Sjöstrand in [S1]. To formulate our result, we need first to introduce that algebra.

\section{§1.2. Sjöstrand algebra of pseudodifferential operators}

In [S1] and [S2], J. Sjöstrand introduced a Wiener-type algebra of pseudodifferential operators as follows. Let $\mathbb{Z}^{2 n}$ be the standard lattice in $\mathbb{R}_{X}^{2 n}$ and let $1=\sum_{j \in \mathbb{Z}^{2 n}} \chi_{0}(X-j), \chi_{0} \in C_{\mathrm{c}}^{\infty}\left(\mathbb{R}^{2 n}\right)$, be a partition of unity. We note $\chi_{j}(X)=\chi_{0}(X-j)$.

Proposition 1.2.1. Let a be a tempered distribution on $\mathbb{R}^{2 n}$. We shall say that a belongs to the class $\mathcal{A}$ if $\omega_{a} \in L^{1}\left(\mathbb{R}^{2 n}\right)$, with $\omega_{a}(\Xi)=\sup _{j \in \mathbb{Z}^{2 n}}$ $\left|\mathcal{F}\left(\chi_{j} a\right)(\Xi)\right|$, where $\mathcal{F}$ is the Fourier transform ${ }^{2}$. Moreover, we have

$$
S_{0,0}^{0} \subset S_{0,0 ; 2 n+1}^{0} \subset \mathcal{A} \subset C^{0}\left(\mathbb{R}^{2 n}\right) \cap L^{\infty}\left(\mathbb{R}^{2 n}\right),
$$

where $S_{0,0 ; 2 n+1}$ is the set of functions defined on $\mathbb{R}^{2 n}$ such that $\left|\left(\partial_{\xi}^{\alpha} \partial_{x}^{\beta} a\right)(x, \xi)\right| \leq C_{\alpha \beta}$ for $|\alpha|+|\beta| \leq 2 n+1$. $\mathcal{A}$ is a Banach algebra for the multiplication with the norm $\|a\|_{\mathcal{A}}=\left\|\omega_{a}\right\|_{L^{1}\left(\mathbb{R}^{2 n}\right)}$.

\footnotetext{
${ }^{2}(\mathcal{F} a)(\Xi)=\int e^{-2 i \pi X \Xi} a(X) d X$. We use also the notation $D_{X_{j}}=\frac{1}{2 i \pi} \partial_{X_{j}}$, so that $\mathcal{F}\left(D^{\alpha} a\right)=\Xi^{\alpha} \mathcal{F} a$.
} 
Proof. In fact, we have the implications $a \in \mathcal{A} \Longrightarrow \mathcal{F}\left(\chi_{j} a\right) \in L^{1}\left(\mathbb{R}^{2 n}\right) \Longrightarrow$ $\chi_{j} a \in C^{0} \cap L^{\infty}$, and, since the sum is locally finite with a fixed overlap ${ }^{3}$, we get $a \in C^{0} \cap L^{\infty}$. Moreover, if $a \in S_{0,0 ; 2 n+1}^{0}$, i.e. is bounded as well as all its derivatives of order $\leq 2 n+1$, we have, with $P(\Xi)=\left(1+\|\Xi\|^{2}\right)^{n}$ the formula $\mathcal{F}\left(\chi_{j} a\right)(\Xi)=P(\Xi)^{-1} \mathcal{F}\left(P\left(D_{X}\right)\left(\chi_{j} a\right)\right)$. We get the identity

$$
\mathcal{F}\left(\chi_{j} a\right)(\Xi)=P(\Xi)^{-1}\left(\Xi_{1}+i\right)^{-1} \mathcal{F}\left(\left(D_{X_{1}}+i\right) P\left(D_{X}\right)\left(\chi_{j} a\right)\right) .
$$

This entails, in the cone $\left\{\Xi \in \mathbb{R}^{2 n}, 2 n\left|\Xi_{1}\right| \geq\|\Xi\|\right\}$ and thus everywhere ${ }^{4}$

$$
\left|\mathcal{F}\left(\chi_{j} a\right)(\Xi)\right| \leq \underbrace{P(\Xi)^{-1}(1+\|\Xi\|)^{-1}}_{\in L^{1}\left(\mathbb{R}^{2 n}\right)} \operatorname{mes}\left(\operatorname{supp} \chi_{0}\right) \sup _{0 \leq k \leq 2 n+1}\left\|a^{(l)}\right\|_{L^{\infty}} C_{n},
$$

yielding the result.

Remark 1.2.2. Since $1 \in \mathcal{A}, \mathcal{A}$ is not included in $\mathcal{F}\left(L^{1}\left(\mathbb{R}^{2 n}\right)\right)$. Moreover $\mathcal{A}$ contains $\mathcal{F}\left(L^{1}\right)$ : let $a$ be a function in $\mathcal{F}\left(L^{1}\right)$. With the above notations, we have

$$
\left|\mathcal{F}\left(\chi_{j} a\right)(\Xi)\right|=\left|\int \hat{\chi}_{0}(\Xi-N) \hat{a}(N) e^{2 i \pi j(N-\Xi)} d N\right| \leq \int\left|\hat{\chi}_{0}(\Xi-N)\right||\hat{a}(N)| d N,
$$

and thus $\int\left|\omega_{a}(\Xi)\right| d \Xi \leq\|\hat{a}\|_{L^{1}}\left\|\widehat{\chi_{0}}\right\|_{L^{1}}$, which gives the inclusion. Moreover, $\mathcal{A}$ is a Banach commutative algebra for the multiplication.

Proposition 1.2.3. The algebra $\mathcal{A}$ is stable by change of quantization, i.e. for all $t$ real, $a \in \mathcal{A} \Longleftrightarrow J^{t} a=\exp \left(2 i \pi t D_{x} \cdot D_{\xi}\right) a \in \mathcal{A}$. The bilinear map $a_{1}, a_{2} \mapsto a_{1} \sharp a_{2}$ is defined on $\mathcal{A} \times \mathcal{A}$ and continuous valued in $\mathcal{A}$, which is a (noncommutative) Banach algebra for $\sharp$. The maps $a \mapsto a^{w}, a(x, D)$ are continuous from $\mathcal{A}$ to $\mathcal{L}\left(L^{2}\left(\mathbb{R}^{n}\right)\right)$.

The proof is given in [S1]. A.Boulkhemair established a lot more results on this algebra in his paper [B1]. In our Appendix A.2, we give a few more properties of the algebra $\mathcal{A}$, which will be useful later on in this article.

We recall that $\left(a_{1} \sharp a_{2}\right)^{w}=a_{1}^{w} a_{2}^{w}$ with

$$
\left(a_{1} \sharp a_{2}\right)(X)=2^{2 n} \iint_{\mathbb{R}^{2 n} \times \mathbb{R}^{2 n}} a_{1}\left(Y_{1}\right) a_{2}\left(Y_{2}\right) e^{-4 i \pi\left[X-Y_{1}, X-Y_{2}\right]} d Y_{1} d Y_{2},
$$

where the bracket [, ] stands for the symplectic form: for $X=(x, \xi), Y=$ $(y, \eta) \in \mathbb{R}^{n} \times \mathbb{R}^{n}$, we have $[X, Y]=\langle\xi, y\rangle-\langle\eta, x\rangle$.

\footnotetext{
${ }^{3}$ If $\cap_{j \in J} \operatorname{supp} \chi_{j} \neq \emptyset$ then card $J \leq N_{0}$, where $N_{0}$ depends only on the compact set $\operatorname{supp} \chi_{0}$.

${ }^{4} \mathbb{R}^{2 n}=\cup_{1 \leq k \leq 2 n}\left\{\Xi \in \mathbb{R}^{2 n}, 2 n\left|\Xi_{k}\right| \geq\|\Xi\|\right\}$ since the complement of that union is empty: it is not possible to find $\Xi$ so that $\max _{1 \leq k \leq 2 n} 2 n\left|\Xi_{k}\right|<\|\Xi\| \leq 2 n \max _{1 \leq k \leq 2 n}\left|\Xi_{k}\right|$.
} 
Comments on the Wiener Lemma. The standard Wiener's Lemma states that if $a \in \ell^{1}\left(\mathbb{Z}^{d}\right)$ is such that $u \mapsto a * u=C_{a} u$ is invertible as an operator on $\ell^{2}\left(\mathbb{Z}^{d}\right)$, then the inverse operator is of the form $C_{b}$ for some $b \in \ell^{1}\left(\mathbb{Z}^{d}\right)$. In [S2] the author is proving several types of Wiener lemma for $\mathcal{A}$. First a commutative version, saying that if $a \in \mathcal{A}$ and $1 / a$ is a bounded function, then $1 / a$ belongs to $\mathcal{A}$. Next, Theorem 4.1 of [S2] provides a noncommutative version of the Wiener lemma for the algebra $\mathcal{A}$ : if an operator $a^{w}$ with $a \in \mathcal{A}$ is invertible as a continuous operator on $L^{2}$, then the inverse operator is $b^{w}$ with $b \in \mathcal{A}$. In the paper [GL], K. Gröchenig and M. Leinert prove several versions of the noncommutative Wiener lemma, and their definition of the twisted convolution ((1.1) in [GL]) is indeed very close to (a discrete version of) the composition formula (1.2.2) above. It would be interesting to compare the methods used to prove these noncommutative versions of the Wiener lemma in the papers [GL] and [S2].

Back to the Gårding inequalities. Also J. Sjöstrand proved in Proposition 5.1 of [S2] the standard Gårding inequality with gain of one derivative for his class, in the semi-classical setting, where $h$ is a small parameter in $(0,1]$ :

$$
a \geq 0, a^{\prime \prime} \in \mathcal{A} \Longrightarrow a(x, h \xi)^{w}+C h \geq 0 .
$$

A consequence of the result (1.1.5) of [Bo1] is that ${ }^{5}$

$$
a \geq 0, a^{(4)} \in S_{0,0}^{0} \Longrightarrow a(x, h \xi)^{w}+C h^{2} \geq 0
$$

Let us ask our second question. Is it possible to get an inequality with gain of 2 derivatives as in (1.2.4) and also to generalize Bony's result by replacing $S_{0,0}^{0}$ by $\mathcal{A}$ ? That would mean that

$$
a \geq 0, a^{(4)} \in \mathcal{A} \Longrightarrow a(x, h \xi)^{w}+C h^{2} \geq 0 .
$$

From the first two inclusions in (1.2.1), we see that (1.2.5) implies (1.2.4). Moreover the constant $C$ in (1.2.5) will depend only on the dimension and on the norm of $a^{(4)}$ in $\mathcal{A}$, which is much more precise than the dependence of $C$ in (1.2.4), which depends on a finite number of semi-norms of $a$ in $S_{0,0}^{0}$. Although (1.2.5) looks stronger than (1.2.3) since $h^{2} \ll h$, it is not obvious to actually deduce (1.2.3) from (1.2.5). Anyhow we shall see that they are both true and that the proof of (1.2.3) is an immediate consequence of the

\footnotetext{
${ }^{5}$ In fact the operator $h^{-2} a(x, h \xi)^{w}$ is unitarily equivalent to $h^{-2} a\left(h^{1 / 2} x, h^{1 / 2} \xi\right)^{w}$ and the function $b(x, \xi)=h^{-2} a\left(h^{1 / 2} x, h^{1 / 2} \xi\right)$ is nonnegative and satisfies $b^{(4)}(x, \xi)=$ $a^{(4)}\left(h^{1 / 2} x, h^{1 / 2} \xi\right)$ which is uniformly in $S_{0,0}^{0}$ whenever $h$ is bounded and $a^{(4)} \in S_{0,0}^{0}$.
} 
most elementary properties of the so-called Wick quantization exposed in our Section 2. Note also that a version of the Hörmander-Melin inequality with gain of $6 / 5$ of derivatives was given, in the semi-classical setting, by F. Hérau in [Hé]: this author used the assumption (6.4) of Theorem 6.2 of [H1], but with a limited regularity on the symbol $a$, which is only such that $a^{(3)} \in \mathcal{A}$.

\section{$\S 1.3$. The main result}

We can state our main result.

Theorem 1.3.1. $\quad$ Let $n$ be a positive integer. There exists a constant $C_{n}$ such that, for all nonnegative functions a defined on $\mathbb{R}^{2 n}$ satisfying $a^{(4)} \in \mathcal{A}$, the operator $a^{w}$ is semi-bounded from below and, more precisely, satisfies

$$
a^{w}+C_{n}\left\|a^{(4)}\right\|_{\mathcal{A}} \geq 0
$$

The Banach algebra $\mathcal{A}$ is defined in Proposition 1.2.1. Note that the constant $C_{n}$ depends only on the dimension $n$.

The proof is given in Section 3.2.

Corollary 1.3.2. Let $n$ be a positive integer.

(i) Let $a(x, \xi)$ be a nonnegative function defined on $\mathbb{R}^{n} \times \mathbb{R}^{n}$ such that (1.1.1) is satisfied for $|\alpha|+|\beta| \leq 2 n+5$. Then (1.1.2) and (1.1.3) hold with a constant $C$ depending only on $n$ and on $\max _{|\alpha|+|\beta| \leq 2 n+5} C_{\alpha \beta}$.

(ii) Let $a(x, \xi, h)$ be a nonnegative function defined on $\mathbb{R}^{n} \times \mathbb{R}^{n} \times(0,1]$ such that

$$
\left|\left(\partial_{\xi}^{\alpha} \partial_{x}^{\beta} a\right)(x, \xi, h)\right| \leq h^{|\alpha|} C_{\alpha \beta}, \quad \text { for } 4 \leq|\alpha|+|\beta| \leq 2 n+5 .
$$

Then $a^{w}+C h^{2} \geq 0$ and $\operatorname{Re} a(x, D)+C h^{2} \geq 0$ hold with a constant $C$ depending only on $n$ and on $\max _{4 \leq|\alpha|+|\beta| \leq 2 n+5} C_{\alpha \beta}$.

(iii) Let $a(x, \xi)$ be a nonnegative function defined on $\mathbb{R}^{n} \times \mathbb{R}^{n}$ such that a ${ }^{(4)}$ belong to $\mathcal{A}$. Then $a(x, h \xi)^{w}+C\left\|a^{(4)}\right\|_{\mathcal{A}} h^{2} \geq 0$ and $\operatorname{Re} a(x, h D)+C\left\|a^{(4)}\right\|_{\mathcal{A}} h^{2} \geq$ 0 hold with a constant $C$ depending only on $n$.

(iv) Let $a(x, \xi, h)$ be a nonnegative function defined on $\mathbb{R}^{n} \times \mathbb{R}^{n} \times(0,1]$ such that, for $|\alpha|+|\beta|=4$, the functions $(x, \xi) \mapsto\left(\partial_{1}^{\beta} \partial_{2}^{\alpha} a\right)\left(x h^{1 / 2}, \xi h^{-1 / 2}, h\right) h^{-|\alpha|}$ belong to $\mathcal{A}$ with a norm bounded above by $\nu_{0}$ for all $h \in(0,1]$. Then $a^{w}+$ $C \nu_{0} h^{2} \geq 0$ and $\operatorname{Re} a(x, D)+C \nu_{0} h^{2} \geq 0$ hold with a constant $C$ depending only on $n$.

That corollary is proven in Section 3.3. 
Remark. It is possible to lower the requirement on the number of derivatives down to $2 n+4+\epsilon$ (any positive $\epsilon$ ) in the statements above, by using conditions on some fractional derivatives as in Theorem 1.1 of [B2].

\section{$\S 2$. The Wick Calculus of Pseudodifferential Operators}

\section{$\S 2.1$. Definitions}

We recall here some facts on the so-called Wick quantization (see e.g. [L1]). That tool was introduced by F. A. Berezin in [Be], and used by many authors. In particular its role and effectiveness in proving the Gårding inequality with gain of one derivative (once called sharp Gårding inequality) was highlighted by the papers of A. Córdoba and C. Fefferman [CF] and A. Unterberger [Un].

Definition 2.1.1. Let $Y=(y, \eta)$ be a point in $\mathbb{R}^{n} \times \mathbb{R}^{n}$.

(i) The operator $\Sigma_{Y}$ is defined as $\left[2^{n} e^{-2 \pi|\cdot-Y|^{2}}\right]^{w}$. This is a rank-one orthogonal projection: $\Sigma_{Y} u=(W u)(Y) \tau_{Y} \varphi$ with $(W u)(Y)=\left\langle u, \tau_{Y} \varphi\right\rangle_{L^{2}\left(\mathbb{R}^{n}\right)}$, where $\varphi(x)=2^{n / 4} e^{-\pi|x|^{2}}$ and $\left(\tau_{y, \eta} \varphi\right)(x)=\varphi(x-y) e^{2 i \pi\left\langle x-\frac{y}{2}, \eta\right\rangle}$.

(ii) Let $a$ be in $L^{\infty}\left(\mathbb{R}^{2 n}\right)$. The Wick quantization of $a$ is defined as

$$
a^{\mathrm{Wick}}=\int_{\mathbb{R}^{2 n}} a(Y) \Sigma_{Y} d Y .
$$

(iii) Let $m$ be a real number. We define $S^{m}$ as the set of smooth functions $p(X, \Lambda)$ defined on $\mathbb{R}^{2 n} \times[1,+\infty)$ such that, for all $k \in \mathbb{N}$,

$$
\sup _{\Lambda \geq 1, X \in \mathbb{R}^{2 n}}\left|\left(\partial_{X}^{k} p\right)(X, \Lambda) \Lambda^{-m+\frac{k}{2}}\right|=\gamma_{k}(p)<\infty .
$$

The following proposition is classical and easy (see e.g. Section 5 in [L1]).

\section{Proposition 2.1.2.}

(i) Let a be in $L^{\infty}\left(\mathbb{R}^{2 n}\right)$. Then $a^{\text {Wick }}=W^{*} a^{\mu} W$ and $1^{\text {Wick }}=I d_{L^{2}\left(\mathbb{R}^{n}\right)}$ where $W$ is the isometric mapping from $L^{2}\left(\mathbb{R}^{n}\right)$ to $L^{2}\left(\mathbb{R}^{2 n}\right)$ given above, and $a^{\mu}$ the operator of multiplication by a in $L^{2}\left(\mathbb{R}^{2 n}\right)$. The operator $\pi_{H}=W W^{*}$ is the orthogonal projection on a closed proper subspace $H$ of $L^{2}\left(\mathbb{R}^{2 n}\right)$. Moreover, we have

$$
\begin{gathered}
\left\|a^{\text {Wick }}\right\|_{\mathcal{L}\left(L^{2}\left(\mathbb{R}^{n}\right)\right)} \leq\|a\|_{L^{\infty}\left(\mathbb{R}^{2 n}\right)}, \\
a(X) \geq 0 \quad \text { for all } X \text { implies } a^{\text {Wick }} \geq 0 .
\end{gathered}
$$


(ii) Let $m$ be a real number and $p \in S^{m}$. Then $p^{\text {Wick }}=p^{w}+r(p)^{w}$, with $r(p) \in S^{m-1}$ so that the mapping $p \mapsto r(p)$ is continuous. More precisely, one has

$$
r(p)(X)=\int_{0}^{1} \int_{\mathbb{R}^{2 n}}(1-\theta) p^{\prime \prime}(X+\theta Y) Y^{2} e^{-2 \pi|Y|^{2}} 2^{n} d Y d \theta .
$$

Note that $r(p)=0$ if $p$ is affine.

(iii) For $a \in L^{\infty}\left(\mathbb{R}^{2 n}\right)$, the Weyl symbol of $a^{\text {Wick }}$ is

$a * 2^{n} \exp -2 \pi|\cdot|^{2} \quad$ which belongs to $S_{0,0}^{0}$ with $k^{\text {th }}$-seminorm $c(k)\|a\|_{L^{\infty}}$.

(iv) With the operator $\Sigma_{Y}$ given in Definition 2.1.1, we have the estimate

$$
\left\|\Sigma_{Y} \Sigma_{Z}\right\|_{\mathcal{L}\left(L^{2}\left(\mathbb{R}^{n}\right)\right)} \leq 2^{n} e^{-\frac{\pi}{2}|Y-Z|^{2}} .
$$

(v) More precisely, the Weyl symbol of $\Sigma_{Y} \Sigma_{Z}$ is, as a function of the variable $X \in \mathbb{R}^{2 n}$,

$$
e^{-\frac{\pi}{2}|Y-Z|^{2}} e^{-2 i \pi[X-Y, X-Z]} 2^{n} e^{-2 \pi\left|X-\frac{Y+Z}{2}\right|^{2}} .
$$

Proposition 2.1.2 is sufficient to prove the standard Gårding inequality with gain of one derivative, and in fact the following improvement was given by J. Sjöstrand in [S2].

Theorem 2.1.3. $\quad$ Let a be a nonnegative function defined on $\mathbb{R}^{2 n}$ such that the second derivatives $a^{\prime \prime}$ belongs to $\mathcal{A}$. Then we have

$$
a^{w}+C_{n}\left\|a^{\prime \prime}\right\|_{\mathcal{A}} \geq 0
$$

Proof. Although a proof of this result is given in [S2] (Proposition 5.1), it is a nice and simple introduction to our more complicated argument of Section 3. From Proposition 2.1.2, we have

$$
a^{w}=a^{\mathrm{Wick}}-r(a)^{w} \geq-r(a)^{w},
$$

with $r(a)(X)=\int_{0}^{1} \int_{\mathbb{R}^{2 n}}(1-\theta) a^{\prime \prime}(X+\theta Y) Y^{2} e^{-2 \pi|Y|^{2}} 2^{n} d Y d \theta$. Since $\mathcal{A}$ is stable by translation (see Lemma A.2.1), we see that $r(a) \in \mathcal{A}$ and thus $r(a)^{w}$ is bounded on $L^{2}\left(\mathbb{R}^{n}\right)$ from Proposition 1.2.3. 
Remark 2.1.4. This theorem implies as well the following semi-classical version; let $a$ be function satisfying the assumption of Theorem 2.1.3. For $h \in$ $(0,1]$, we define $A_{h}(x, \xi)=h^{-1} a\left(x h^{1 / 2}, \xi h^{1 / 2}\right)$. The function $A_{h}$ is nonnegative with a second derivative bounded in $\mathcal{A}$ by $\operatorname{cst} \times\left\|a^{\prime \prime}\right\|_{\mathcal{A}}$ (see Lemma A.2.1), so that the previous theorem implies, with $C$ depending only on the dimension, that $A_{h}^{w}+C\left\|a^{\prime \prime}\right\|_{\mathcal{A}} \geq 0$. Since $A_{h}^{w}$ is unitarily equivalent to $h^{-1} a(x, h \xi)^{w}$, this gives

$$
a(x, h \xi)^{w}+h C\left\|a^{\prime \prime}\right\|_{\mathcal{A}} \geq 0 .
$$

\section{$\S 2.2$. Sharp estimates for the remainders}

Proposition 2.1.2 falls short of providing a proof for the Fefferman-Phong inequality, which gains two derivatives.

Lemma 2.2.1. $\quad$ Let a be a function defined on $\mathbb{R}^{2 n}$ such that the fourth derivatives $a^{(4)}$ belong to $\mathcal{A}$. Then we have

$$
a^{w}=\left(a-\frac{1}{8 \pi} \operatorname{trace} a^{\prime \prime}\right)^{W i c k}+\rho_{0}\left(a^{(4)}\right)^{w}
$$

with $\rho_{0}\left(a^{(4)}\right) \in \mathcal{A}$ and more precisely $\left\|\rho_{0}\left(a^{(4)}\right)\right\|_{\mathcal{A}} \leq C_{n}\left\|a^{(4)}\right\|_{\mathcal{A}}$.

Proof. The Weyl symbol $\sigma_{a}$ of $a^{\text {Wick }}$ is

$$
\begin{aligned}
\sigma_{a}(X) & =\int a(X+Y) 2^{n} e^{-2 \pi|Y|^{2}} d Y \\
& =a(X)+\int \frac{1}{2} a^{\prime \prime}(X) Y^{2} 2^{n} e^{-2 \pi|Y|^{2}} d Y \\
& +\frac{1}{3 !} \iint_{0}^{1}(1-\theta)^{3} a^{(4)}(X+\theta Y) Y^{4} 2^{n} e^{-2 \pi|Y|^{2}} d Y d \theta \\
& =a(X)+\frac{1}{8 \pi} \operatorname{trace} a^{\prime \prime}(X) \\
& +\frac{1}{3 !} \iint_{0}^{1}(1-\theta)^{3} a^{(4)}(X+\theta Y) Y^{4} 2^{n} e^{-2 \pi|Y|^{2}} d Y d \theta .
\end{aligned}
$$

Moreover the Weyl symbol $\theta_{a}$ of $\left(\operatorname{trace} a^{\prime \prime}\right)^{\text {Wick }}$ is, from Proposition 2.1.2,

$$
\theta_{a}(X)=\operatorname{trace} a^{\prime \prime}(X)+\int_{0}^{1} \int_{\mathbb{R}^{2 n}}(1-\theta)\left(\operatorname{trace} a^{\prime \prime}\right)^{\prime \prime}(X+\theta Y) Y^{2} e^{-2 \pi|Y|^{2}} 2^{n} d Y d \theta
$$


As a result, the Weyl symbol of the operator $\left(a-\frac{1}{8 \pi} \text { trace } a^{\prime \prime}\right)^{\text {Wick }}$ is

$$
\begin{aligned}
& a+\frac{1}{3 !} \iint_{0}^{1}(1-\theta)^{3} a^{(4)}(X+\theta Y) Y^{4} 2^{n} e^{-2 \pi|Y|^{2}} d Y d \theta \\
& -\frac{1}{8 \pi} \int_{0}^{1} \int_{\mathbb{R}^{2 n}}(1-\theta)\left(\operatorname{trace} a^{\prime \prime}\right)^{\prime \prime}(X+\theta Y) Y^{2} e^{-2 \pi|Y|^{2}} 2^{n} d Y d \theta .
\end{aligned}
$$

We get the equality in the lemma with

$$
\begin{aligned}
\rho_{0}\left(a^{(4)}\right)(X) & =\frac{1}{8 \pi} \int_{0}^{1} \int_{\mathbb{R}^{2 n}}(1-\theta)\left(\operatorname{trace} a^{\prime \prime}\right)^{\prime \prime}(X+\theta Y) Y^{2} e^{-2 \pi|Y|^{2}} 2^{n} d Y d \theta \\
& -\frac{1}{3 !} \iint_{0}^{1}(1-\theta)^{3} a^{(4)}(X+\theta Y) Y^{4} 2^{n} e^{-2 \pi|Y|^{2}} d Y d \theta
\end{aligned}
$$

We note now that $\rho_{0}$ depends linearly on $a^{(4)}$ and that

$$
\rho_{0}\left(a^{(4)}\right)(X)=\iint_{0}^{1} a^{(4)}(X+\theta Y) \underbrace{M(\theta, Y)}_{\substack{\text { polynomial } \\ \text { in } Y, \theta .}} e^{-2 \pi|Y|^{2}} d Y d \theta .
$$

Looking now at the formula (2.2.2) and applying Lemma A.2.1, we get

$$
\left\|\rho_{0}\left(a^{(4)}\right)\right\|_{\mathcal{A}} \leq \iint_{0}^{1} M(\theta, Y) e^{-2 \pi|Y|^{2}} d Y d \theta C_{0}\left\|a^{(4)}\right\|_{\mathcal{A}}=C_{1}\left\|a^{(4)}\right\|_{\mathcal{A}} .
$$

The proof of Lemma 2.2.1 is complete.

Remark 2.2.2. We note that, from Lemma 2.2.1 and the $L^{2}$ boundedness of operators with symbols in $\mathcal{A}$, Theorem 1.3.1 is reduced to proving

$$
a \geq 0, a^{(4)} \in \mathcal{A} \Longrightarrow\left(a-\frac{1}{8 \pi} \text { trace } a^{\prime \prime}\right)^{\text {Wick }} \text { is semi-bounded from below. }
$$

Naturally, one should not expect the quantity $a-\frac{1}{8 \pi}$ trace $a^{\prime \prime}$ to be nonnegative: this quantity will take negative values even in the simplest case $a(x, \xi)=x^{2}+\xi^{2}$, so that the positivity of the quantization expressed by (2.1.3) is far from enough to get our result. We shall prove in Section 3 a stronger version of (2.2.3), but before this, we need to investigate more closely the composition formula for the Wick quantization. 


\section{§2.3. On the composition formula for the Wick quantization}

In this section, we prove some formulas of composition for operators with very irregular Wick symbols. The first lemma below was already proven in [L1], but we give here a complete proof for the convenience of the reader, since these (easy) computations are not completely standard.

Lemma 2.3.1. For $p, q \in L^{\infty}\left(\mathbb{R}^{2 n}\right)$ real-valued with $p^{\prime \prime} \in L^{\infty}\left(\mathbb{R}^{2 n}\right)$, we have

$$
\begin{aligned}
\operatorname{Re}\left(p^{\text {Wick }} q^{\text {Wick }}\right) & =\left(p q-\frac{1}{4 \pi} \nabla p \cdot \nabla q\right)^{\text {Wick }}+R, \\
\|R\|_{\mathcal{L}\left(L^{2}\left(\mathbb{R}^{n}\right)\right)} & \leq C(n)\left\|p^{\prime \prime}\right\|_{L^{\infty}}\|q\|_{L^{\infty}} .
\end{aligned}
$$

The product $\nabla p \cdot \nabla q$ above makes sense (see our Appendix A.3) as a tempered distribution since $\nabla p$ is a Lipschitz continuous function and $\nabla q$ is the derivative of an $L^{\infty}$ function: in fact, we shall use as a definition (see our Appendix A.3) $\nabla p \cdot \nabla q=\nabla \cdot(\underbrace{q}_{L^{\infty}} \underbrace{\nabla p}_{\text {Lip. }})-\underbrace{q}_{L^{\infty}} \underbrace{\Delta p}_{L^{\infty}}$.

Proof. Using Definition 2.1.1, we see that

$$
\begin{aligned}
p^{\mathrm{Wick}} q^{\mathrm{Wick}} & =\iint_{\mathbb{R}^{2 n} \times \mathbb{R}^{2 n}} p(Y) q(Z) \Sigma_{Y} \Sigma_{Z} d Y d Z \\
& =\iint\left(p(Z)+p^{\prime}(Z)(Y-Z)+p_{2}(Z, Y)(Y-Z)^{2}\right) q(Z) \Sigma_{Y} \Sigma_{Z} d Y d Z \\
& =\int(p q)(Z) \Sigma_{Z} d Z+\iint p^{\prime}(Z)(Y-Z) \Sigma_{Y} d Y q(Z) \Sigma_{Z} d Z+R_{0}, \\
& \text { with } R_{0}=\iiint_{0}^{1}(1-\theta) p^{\prime \prime}(Z+\theta(Y-Z))(Y-Z)^{2} q(Z) \Sigma_{Y} \Sigma_{Z} d Y d Z d \theta .
\end{aligned}
$$

Claim 2.3.2. Let $\omega$ be a measurable function defined on $\mathbb{R}^{2 n} \times \mathbb{R}^{2 n}$ such that

$$
|\omega(Y, Z)| \leq \gamma_{0}(1+|Y-Z|)^{N_{0}} .
$$

Then the operator $\iint \omega(Y, Z) \Sigma_{Y} \Sigma_{Z} d Y d Z$ is bounded on $L^{2}\left(\mathbb{R}^{n}\right)$ with $\mathcal{L}\left(L^{2}\left(\mathbb{R}^{n}\right)\right)$ norm bounded above by a constant depending on $\gamma_{0}, N_{0}$. This is an immediate consequence of Cotlar's lemma (see e.g. Lemma 4.2 .3 in [BL] or Lemma 18.6.5 in [H2]) and of the formula (2.1.6).

Using that claim, we obtain that

$$
\left\|R_{0}\right\|_{\mathcal{L}\left(L^{2}\left(\mathbb{R}^{n}\right)\right)} \leq C_{1}(n)\left\|p^{\prime \prime}\right\|_{L^{\infty}\left(\mathbb{R}^{2 n}\right)}\|q\|_{L^{\infty}\left(\mathbb{R}^{2 n}\right)}
$$


We check now $\int(Y-Z) \Sigma_{Y} d Y$ whose Weyl symbol is, as a function of $X$,

$$
\int(Y-Z) 2^{n} e^{-2 \pi|X-Y|^{2}} d Y=\int(X-Z) 2^{n} e^{-2 \pi|X-Y|^{2}} d Y=X-Z
$$

So with $L_{Z}(X)=X-Z$, we have $\int(Y-Z) \Sigma_{Y} d Y \Sigma_{Z}=(X-Z)^{w} \Sigma_{Z}=L_{Z}^{w} \Sigma_{Z}$ and thus

$$
\begin{aligned}
\operatorname{Re} \int(Y-Z) \Sigma_{Y} d Y \Sigma_{Z} & =\operatorname{Re}\left(L_{Z}^{w} \Sigma_{Z}\right)=\left((X-Z) 2^{n} e^{-2 \pi|X-Z|^{2}}\right)^{w} \\
& =\frac{1}{4 \pi} \partial_{Z}\left(2^{n} e^{-2 \pi|X-Z|^{2}}\right)^{w}
\end{aligned}
$$

so that

$$
\operatorname{Re} \int(Y-Z) \Sigma_{Y} d Y \Sigma_{Z}=\frac{1}{4 \pi} \partial_{Z}\left(\Sigma_{Z}\right)
$$

Using that $p$ and $q$ are real-valued, the formula for $\operatorname{Re}\left(p^{\text {Wick }} q^{\text {Wick }}\right)$ becomes

$$
\begin{aligned}
\operatorname{Re}\left(p^{\text {Wick }} q^{\text {Wick }}\right) & =\int(p q)(Z) \Sigma_{Z} d Z+\int p^{\prime}(Z) q(Z) \frac{1}{4 \pi} \partial_{Z} \Sigma_{Z} d Z+\operatorname{Re} R_{0} \\
& =\int\left((p q)(Z)-\frac{1}{4 \pi} p^{\prime}(Z) \cdot q^{\prime}(Z)\right) \Sigma_{Z} d Z \\
& -\int \frac{1}{4 \pi} \operatorname{trace} p^{\prime \prime}(Z) q(Z) \Sigma_{Z} d Z+\operatorname{Re} R_{0}
\end{aligned}
$$

that is the result of the lemma, using (2.3.1) and (2.1.2) for the penultimate term on the line above.

The next lemma is more involved.

Lemma 2.3.3. For $p$ measurable real-valued function such that $p^{\prime \prime}$, $\left(p^{\prime} p^{\prime \prime}\right)^{\prime},\left(p p^{\prime \prime}\right)^{\prime \prime} \in L^{\infty}$, we have

$$
\begin{gathered}
p^{W i c k} p^{W i c k}=\int\left[p(Z)^{2}-\frac{1}{4 \pi}|\nabla p(Z)|^{2}\right] \Sigma_{Z} d Z+S \\
\|S\|_{\mathcal{L}\left(L^{2}\left(\mathbb{R}^{n}\right)\right)} \leq C(n)\left(\left\|p^{\prime \prime}\right\|_{L^{\infty}}^{2}+\left\|\left(p^{\prime \prime} p^{\prime}\right)^{\prime}\right\|_{L^{\infty}}+\left\|\left(p p^{\prime \prime}\right)^{\prime \prime}\right\|_{L^{\infty}}\right) .
\end{gathered}
$$

Here $p^{\prime \prime}$ stands for the vector (tensor) with components $\left(\partial_{X}^{\alpha} p\right)_{|\alpha|=2}$, whereas the components of $\left(p^{\prime \prime} p^{\prime}\right)^{\prime}$ are $\partial_{X}^{\alpha}\left(\partial_{X}^{\beta} \partial_{X}^{\gamma} p\right)_{\substack{|\alpha|=1,|\beta|=2 \\|\gamma|=1}}$, and those of $\left(p p^{\prime \prime}\right)^{\prime \prime}$ are $\partial_{X}^{\alpha}\left(p \partial_{X}^{\beta} p\right)_{|\alpha|=|\beta|=2}$. 
Proof. We have

$$
\begin{aligned}
p^{\text {Wick }} p^{\text {Wick }} & =\iint p(Y) p(Z) \Sigma_{Y} \Sigma_{Z} d Y d Z \\
& =\iint p(Y)\left(p(Y)+p^{\prime}(Y)(Z-Y)\right) \Sigma_{Y} \Sigma_{Z} d Y d Z \\
& +\iiint_{0}^{1} p(Y)(1-\theta) p^{\prime \prime}(Y+\theta(Z-Y)) d \theta(Z-Y)^{2} \Sigma_{Y} \Sigma_{Z} d Y d Z d \theta
\end{aligned}
$$

so that, using (2.3.2) for the terms $p p^{\prime}$ in the double integral above, we get, $\operatorname{noting} \operatorname{trace}\left(p^{\prime \prime}\right)=\Delta p$,

$$
p^{\mathrm{Wick}} p^{\mathrm{Wick}}=\left(p^{2}-\frac{1}{4 \pi}|\nabla p|^{2}-\frac{1}{4 \pi} p \Delta p\right)^{\mathrm{Wick}}+\operatorname{Re}\left(\Omega_{0}+\Omega_{1}+\Omega_{2}\right)
$$

with

$\Omega_{0}=\iiint_{0}^{1} p(Y+\theta(Z-Y)) p^{\prime \prime}(Y+\theta(Z-Y))(Z-Y)^{2} \Sigma_{Y} \Sigma_{Z} d Y d Z(1-\theta) d \theta$,

$$
\begin{aligned}
\Omega_{1}=\iiint_{0}^{1} & p^{\prime}(Y+\theta(Z-Y)) \theta(Y-Z) \\
& \times p^{\prime \prime}(Y+\theta(Z-Y))(Z-Y)^{2} \Sigma_{Y} \Sigma_{Z} d Y d Z(1-\theta) d \theta
\end{aligned}
$$

and from the claim (2.3.2),

$$
\left\|\Omega_{2}\right\|_{\mathcal{L}\left(L^{2}\left(\mathbb{R}^{n}\right)\right)} \leq C_{1}(n)\left\|p^{\prime \prime}\right\|_{L^{\infty}}^{2} .
$$

We write now $\Omega_{0}=\Omega_{00}+\Omega_{01}, \Omega_{1}=\Omega_{10}+\Omega_{11}$ with

$$
\begin{gathered}
\Omega_{00}=\frac{1}{2} \iint p(Y) p^{\prime \prime}(Y)(Z-Y)^{2} \Sigma_{Y} \Sigma_{Z} d Y d Z, \\
\Omega_{01}=\iiint_{0}^{1}\left(\left(p p^{\prime \prime}\right)(Y+\theta(Z-Y))-\left(p p^{\prime \prime}\right)(Y)\right)(Z-Y)^{2} \\
\times \Sigma_{Y} \Sigma_{Z} d Y d Z(1-\theta) d \theta \\
\Omega_{10}=-\frac{1}{6} \iiint_{0}^{1} p^{\prime}(Y)(Z-Y) p^{\prime \prime}(Y)(Z-Y)^{2} \Sigma_{Y} \Sigma_{Z} d Y d Z \\
\qquad \Omega_{11}\left\|\mathcal{L}\left(L^{2}\left(\mathbb{R}^{n}\right)\right) \leq C_{2}(n)\right\|\left(p^{\prime} p^{\prime \prime}\right)^{\prime} \|_{L^{\infty}} .
\end{gathered}
$$


We have also $\Omega_{01}=\Omega_{010}+\Omega_{011}$ with

$$
\begin{aligned}
& \Omega_{010}= \frac{1}{6} \iint\left(p p^{\prime \prime}\right)^{\prime}(Y)(Z-Y)(Z-Y)^{2} \Sigma_{Y} \Sigma_{Z} d Y d Z, \\
&\left\|\Omega_{011}\right\|_{\mathcal{L}\left(L^{2}\left(\mathbb{R}^{n}\right)\right)} \leq C_{3}(n)\left\|\left(p p^{\prime \prime}\right)^{\prime \prime}\right\|_{L^{\infty}} .
\end{aligned}
$$

From (2.3.5-10), it suffices to check that the following term is a remainder satisfying the estimate (2.3.4) to get the result of Lemma 2.3.3:

$$
\begin{aligned}
\widetilde{\Omega}= & -\frac{1}{4 \pi} \int p(Y) \operatorname{trace} p^{\prime \prime}(Y) \Sigma_{Y} d Y \\
& +\frac{1}{2} \operatorname{Re} \iint\left(p p^{\prime \prime}\right)(Y)(Z-Y)^{2} \Sigma_{Y} \Sigma_{Z} d Y d Z \\
& +\frac{1}{6} \operatorname{Re} \iint\left(p p^{\prime \prime}\right)^{\prime}(Y)(Z-Y)(Z-Y)^{2} \Sigma_{Y} \Sigma_{Z} d Y d Z \\
& -\frac{1}{6} \operatorname{Re} \iiint_{0}^{1} p^{\prime}(Y)(Z-Y) p^{\prime \prime}(Y)(Z-Y)^{2} \Sigma_{Y} \Sigma_{Z} d Y d Z .
\end{aligned}
$$

The real part of the Weyl symbol of $\int\left(Z_{j}-Y_{j}\right)\left(Z_{k}-Y_{k}\right)\left(Z_{l}-Y_{l}\right) \Sigma_{Y} \Sigma_{Z} d Z$ is (see $(2.1 .7))$

$$
\begin{aligned}
& \int\left(Z_{j}-Y_{j}\right)\left(Z_{k}-Y_{k}\right)\left(Z_{l}-Y_{l}\right) e^{-\frac{\pi}{2}|Y-Z|^{2}} \\
& \quad \times \cos (2 \pi[X-Y, X-Z]) 2^{n} e^{-2 \pi\left|X-\frac{Y+Z}{2}\right|^{2}} d Z \\
& =\int T_{j} T_{k} T_{l} e^{-2 \pi|T / 2|^{2}} \cos (2 \pi[X-Y, T]) 2^{n} e^{-2 \pi\left|X-Y-\frac{T}{2}\right|^{2}} d T \\
& =\int T_{j} T_{k} T_{l} \cos (2 \pi[X-Y, T]) e^{-\pi|X-Y-T|^{2}} d T 2^{n} e^{-\pi|X-Y|^{2}}=\nu_{j k l}(X-Y)
\end{aligned}
$$

with

$$
\begin{aligned}
& \nu_{j k l}(S)= \int T_{j} T_{k} T_{l} \cos (2 \pi[S, T]) e^{-\pi|S-T|^{2}} d T 2^{n} e^{-\pi|S|^{2}} \\
&= 2^{n} e^{-\pi|S|^{2}} \int\left(T_{j}+S_{j}\right)\left(T_{k}+S_{k}\right)\left(T_{l}+S_{l}\right) \cos (2 \pi[S, T]) e^{-\pi|T|^{2}} d T \\
&=2^{n} e^{-\pi|S|^{2}} \int\left(T_{j} T_{k} S_{l}+T_{k} T_{l} S_{j}+T_{l} T_{j} S_{k}\right. \\
&\left.\quad+S_{j} S_{k} S_{l}\right) \cos (2 \pi[S, T]) e^{-\pi|T|^{2}} d T
\end{aligned}
$$


We notice that the function $S \mapsto \int_{\mathbb{R}^{2 n}} T_{j} T_{k} \exp (2 i \pi[S, T]) e^{-\pi|T|^{2}} d T$ is a secondorder derivative of $S \mapsto \int_{\mathbb{R}^{2 n}} \exp (2 i \pi[S, T]) e^{-\pi|T|^{2}} d T=e^{-\pi|S|^{2}}$ so that

$$
2^{n} e^{-\pi|S|^{2}} S_{l} \int_{\mathbb{R}^{2 n}} T_{j} T_{k} \cos (2 \pi[S, T]) e^{-\pi|T|^{2}} d T=e^{-2 \pi|S|^{2}} S_{l} P_{j k}(S),
$$

with $P_{j k}$ even, second-order and real polynomial. The function $S_{l_{1}} S_{l_{2}} S_{l_{3}}$ $\times e^{-2 \pi|S|^{2}}$ is always a linear combination of derivatives of Schwartz functions on $\mathbb{R}^{2 n}$, since

if $l_{1}<l_{2} \leq l_{3}$ it is the derivative with respect to $S_{l_{1}}$ of $S_{l_{2}} S_{l_{3}} e^{-2 \pi|S|^{2}}(-4 \pi)^{-1}$, if $l_{1}=l_{2}<l_{3}$ it is the derivative with respect to $S_{l_{3}}$ of $S_{l_{1}} S_{l_{2}} e^{-2 \pi|S|^{2}}(-4 \pi)^{-1}$,

if $l_{1}=l_{2}=l_{3}=l$ it is a linear combination of the third and first derivative with respect to $S_{l}$ of $e^{-2 \pi|S|^{2}}$, since

$$
\left(e^{t^{2}}\right)^{\prime \prime \prime}=\left(12 t+8 t^{3}\right) e^{t^{2}}, \quad t^{3} e^{t^{2}}=\frac{1}{8}\left(e^{t^{2}}\right)^{\prime \prime \prime}-\frac{3}{4}\left(e^{t^{2}}\right)^{\prime} .
$$

As a result the function $\nu_{j k l}$ defined by (2.3.12) is a linear combination of derivatives with respect to $S_{j}, S_{k}$ or $S_{l}$ of Schwartz functions on $\mathbb{R}^{2 n}$. Integrating by parts in the last two terms of (2.3.11), we see that their $\mathcal{L}\left(L^{2}\right)$ norm is bounded from above by $C_{4}(n)\left(\left\|\left(p p^{\prime \prime}\right)^{\prime \prime}\right\|_{L^{\infty}}+\left\|\left(p^{\prime} p^{\prime \prime}\right)^{\prime}\right\|_{L^{\infty}}\right)$. Looking at (2.3.11), we see that we are left with

$\widetilde{\Omega}_{0}=-\frac{1}{4 \pi} \int p(Y) \operatorname{trace} p^{\prime \prime}(Y) \Sigma_{Y} d Y+\frac{1}{2} \operatorname{Re} \iint\left(p p^{\prime \prime}\right)(Y)(Z-Y)^{2} \Sigma_{Y} \Sigma_{Z} d Y d Z$.

The real part of the operator $\int\left(Z_{j}-Y_{j}\right)\left(Z_{k}-Y_{k}\right) \Sigma_{Y} \Sigma_{Z} d Y d Z$ has the Weyl symbol (function of $X$ )

$$
\begin{aligned}
& \int T_{j} T_{k} e^{-\pi|X-Y-T|^{2}} \cos (2 \pi[X-Y, T]) d T 2^{n} e^{-\pi|X-Y|^{2}} \\
& =\int\left(\left(X_{j}-Y_{j}\right)\left(X_{k}-Y_{k}\right)+T_{j} T_{k}\right) e^{-\pi|T|^{2}} \cos (2 \pi[X-Y, T]) d T 2^{n} e^{-\pi|X-Y|^{2}} \\
& =\int\left(S_{j} S_{k}+T_{j} T_{k}\right) e^{-\pi|T|^{2}} \cos (2 \pi[S, T]) d T 2^{n} e^{-\pi|S|^{2}}, \quad S=X-Y .
\end{aligned}
$$

- If $j \neq k$, both terms in (2.3.14) are second order derivatives with respect to $Y$ of a Schwartz function in $\mathbb{R}^{2 n}$. In fact the first term is

$$
S_{j} S_{k} 2^{n} e^{-2 \pi|S|^{2}}=\partial_{S_{j}} \partial_{S_{k}}\left(2^{n} e^{-2 \pi|S|^{2}} / 16 \pi^{2}\right)=\partial_{Y_{j}} \partial_{Y_{k}}\left(2^{n} e^{-2 \pi|S|^{2}} / 16 \pi^{2}\right)
$$


and the second term is equal to $-S_{j^{\prime}} S_{k^{\prime}} 2^{n} e^{-2 \pi|S|^{2}}$, with $j^{\prime} \neq k^{\prime}$, also a secondorder derivative. The contribution of these terms in (2.3.13) is then, after integration by parts, an $L^{2}$ bounded operator with norm $\leq C_{5}(n)\left\|\left(p p^{\prime \prime}\right)^{\prime \prime}\right\|_{L^{\infty}}$.

- If $j=k$, with $j^{\prime}=j \pm n$ (in fact $j^{\prime}=j+n$ if $1 \leq j \leq n$ and $j^{\prime}=j-n$ if $1+n \leq j \leq 2 n)$, we note that $(2.3 .14)$ is equal to

$S_{j}^{2} 2^{n} e^{-2 \pi|S|^{2}}-\frac{1}{4 \pi^{2}} e^{-\pi|S|^{2}} \partial_{S_{j^{\prime}}}^{2}\left(2^{n} e^{-\pi|S|^{2}}\right)=2^{n} e^{-2 \pi|S|^{2}}\left(S_{j}^{2}-\frac{1}{4 \pi^{2}}\left(4 \pi^{2} S_{j^{\prime}}^{2}-2 \pi\right)\right)$.

Taking into account the contribution of these terms in (2.3.13), we see that we are left with

$$
-\frac{1}{4 \pi} \int p(Y) \operatorname{trace} p^{\prime \prime}(Y) \Sigma_{Y} d Y+\frac{1}{2} \iint \frac{1}{2 \pi} \operatorname{trace}\left(p p^{\prime \prime}\right)(Y) \Sigma_{Y} d Y=0 .
$$

The proof of Lemma 2.3.3 is complete.

\section{$\S 3 . \quad$ The Proof}

\section{$\S 3.1$. Nonnegative functions as sum of squares}

Theorem 3.1.1. $\quad$ Let $m$ be a nonnegative integer. There exists an integer $N$ and a positive constant $C$ such that the following property holds. Let a be a nonnegative $C^{3,1}$ function ${ }^{6}$ defined on $\mathbb{R}^{m}$ such that $a^{(4)} \in L^{\infty}$; then we can write

$$
a=\sum_{1 \leq j \leq N} b_{j}^{2}
$$

where the $b_{j}$ are $C^{1,1}$ functions such that $b_{j}^{\prime \prime},\left(b_{j}^{\prime} b_{j}^{\prime \prime}\right)^{\prime},\left(b_{j} b_{j}^{\prime \prime}\right)^{\prime \prime} \in L^{\infty}$. More precisely, we have

$$
\left\|b_{j}^{\prime \prime}\right\|_{L^{\infty}}^{2}+\left\|\left(b_{j}^{\prime} b_{j}^{\prime \prime}\right)^{\prime}\right\|_{L^{\infty}}+\left\|\left(b_{j} b_{j}^{\prime \prime}\right)^{\prime \prime}\right\|_{L^{\infty}} \leq C\left\|a^{(4)}\right\|_{L^{\infty}} .
$$

Note that this implies that each function $b_{j}$ is such that $b_{j}^{2}$ is $C^{3,1}$ and that $N$ and $C$ depend only on the dimension $m$.

Remark 3.1.2. We shall use the following notation: let $A$ be a symmetric $k$-linear form on real normed vector space $V$. We define the norm of $A$ by

$$
\|A\|=\sup _{\|T\|=1}\left|A T^{k}\right| .
$$

\footnotetext{
${ }^{6} \mathrm{~A} C^{3,1}$ function is a $C^{3}$ function whose third-order derivatives are Lipschitz continuous.
} 
Since the symmetrized products of $T_{1} \otimes \cdots \otimes T_{k}$ can be written as a linear combination of $k$-th powers, that norm is equivalent to the natural norm

$$
\|A\|=\sup _{\substack{\left\|T_{j}\right\|=1, 1 \leq j \leq k}}\left|A T_{1} \ldots T_{k}\right|
$$

and in fact, when $V$ is Euclidean, we have the equality $\|A\|=\|A\|$ (see the paper by O. D. Kellogg [Ke]). In our Appendix A.4, we prove that for an arbitrary normed space, the best estimate is $\|A\| \leq \frac{k^{k}}{k !}\|A\|$.

Comment. Part of this theorem is a consequence of the classical proof of the Fefferman-Phong inequality and of the more refined analysis of Bony in [Bo1] (see also [Gu] and [Ta]). However the control of the $L^{\infty}$ norm of the quantities $\left(b_{j}^{\prime} b_{j}^{\prime \prime}\right)^{\prime},\left(b_{j} b_{j}^{\prime \prime}\right)^{\prime \prime}$ is more difficult to achieve and seems to be new. Naturally the inequality (3.1.2) is a key element of our proof, since it is connected with the estimates (2.3.4). We shall thus focus our attention on the new elements of the proof, referring the reader to our appendix or to the literature for the more standard points.

Proof of Theorem 3.1.1. We define

$$
\rho(x)=\left(|a(x)|+\left|a^{\prime \prime}(x)\right|^{2}\right)^{1 / 4}, \quad \Omega=\{x, \rho(x)>0\},
$$

assuming as we may $\left\|a^{(4)}\right\|_{L^{\infty}} \leq 1$. Note that, since $\rho$ is continuous, the set $\Omega$ is open. The metric $|d x|^{2} / \rho(x)^{2}$ is slowly varying in $\Omega$ (see Lemma A.1.2): $\exists r_{0}>0, C_{0} \geq 1$ such that

$$
x \in \Omega,|y-x| \leq r_{0} \rho(x) \Longrightarrow y \in \Omega, C_{0}^{-1} \leq \frac{\rho(x)}{\rho(y)} \leq C_{0} .
$$

The constants $r_{0}, C_{0}$ can be chosen as "universal" constants, thanks to the normalization on $a^{(4)}$ above. Moreover, using Lemma A.1.1, the nonnegativity of $a$ implies with $\gamma_{j}=1$ for $j=0,2,4, \gamma_{1}=3, \gamma_{3}=4$,

$$
\left|a^{(j)}(x)\right| \leq \gamma_{j} \rho(x)^{4-j}, \quad 1 \leq j \leq 4 .
$$

We refer the reader to Section 1.4 in [H2] for the basic properties of slowly varying metrics as well as for the following lemma.

Lemma 3.1.3. Let $a, \rho, \Omega, r_{0}$ be as above. There exists a positive number $r_{0}^{\prime} \leq r_{0}$, such that for all $\left.\left.r \in\right] 0, r_{0}^{\prime}\right]$, there exists a sequence $\left(x_{\nu}\right)_{\nu \in \mathbb{N}}$ of points in $\Omega$ and a positive number $M_{r}$, such that the following properties are satisfied. 
We define $U_{\nu}, U_{\nu}^{*}, U_{\nu}^{* *}$ as the closed Euclidean balls with center $x_{\nu}$ and radius $r \rho_{\nu}, 2 r \rho_{\nu}, 4 r \rho_{\nu}$ with $\rho_{\nu}=\rho\left(x_{\nu}\right)$. There exist two families of nonnegative smooth functions on $\mathbb{R}^{m},\left(\varphi_{\nu}\right)_{\nu \in \mathbb{N}},\left(\psi_{\nu}\right)_{\nu \in \mathbb{N}}$ such that

$$
\sum_{\nu} \varphi_{\nu}^{2}(x)=\mathbf{1}_{\Omega}(x), \operatorname{supp} \varphi_{\nu} \subset U_{\nu}, \quad \psi_{\nu} \equiv 1 \quad \text { on } U_{\nu}^{*}, \operatorname{supp} \psi_{\nu} \subset U_{\nu}^{* *} \subset \Omega .
$$

Moreover, for all integers $l$, we have $\sup _{x \in \Omega, \nu \in \mathbb{N}}\left\|\varphi_{\nu}^{(l)}(x)\right\| \rho_{\nu}^{l}+\sup _{x \in \Omega, \nu \in \mathbb{N}}$ $\times\left\|\psi_{\nu}^{(l)}(x)\right\| \rho_{\nu}^{l}<\infty$. The overlap of the balls $U_{\nu}^{* *}$ is bounded, i.e.

$$
\bigcap_{\nu \in \mathcal{N}} U_{\nu}^{* *} \neq \emptyset \quad \Longrightarrow \quad \# \mathcal{N} \leq M_{r}
$$

Moreover, $\rho(x) \sim \rho_{\nu}$ all over $U_{\nu}^{* *}$ (i.e. the ratios $\rho(x) / \rho_{\nu}$ are bounded above and below by a fixed constant, provided that $\left.x \in U_{\nu}^{* *}\right)$.

Since $a$ is vanishing on $\Omega^{c}$, we obtain

$$
a(x)=\sum_{\nu \in \mathbb{N}} a(x) \varphi_{\nu}^{2}(x) .
$$

Definition 3.1.4. Let $a, \rho, \Omega$ be as above. Let $\theta$ be a positive number $\leq \theta_{0}$, where $\theta_{0}$ is a fixed constant satisfying the requirements of Lemma A.1.5. A point $x \in \Omega$ is said to be

(i) $\theta$-elliptic whenever $a(x) \geq \theta \rho(x)^{4}$,

(ii) $\theta$-nondegenerate whenever $a(x)<\theta \rho(x)^{4}$ : we have then $\left\|a^{\prime \prime}(x)\right\|^{2} \geq$ $\rho(x)^{4} / 2$.

We go on now with the proof of Theorem 3.1.1. We choose a positive number $\theta$ satisfying the condition in Definition 3.1.4. We choose a positive number $r \leq r_{0}^{\prime}$ as defined in Lemma 3.1.3 and we consider a sequence $\left(x_{\nu}\right)$ as in that lemma. We assume also that $4 r \leq \theta / 8$, so that Lemma 3.1 .3 can be applied on the ball $U_{\nu}^{* *}$.

Let us first consider the "elliptic" indices $\nu$ such that $x_{\nu}$ is $\theta$-elliptic. According to Lemma A.1.3, for $x \in U_{\nu}^{* *}$, we have $a(x) \sim \rho_{\nu}^{4}$, so that with

$$
b_{\nu}(x)=a(x)^{1 / 2} \psi_{\nu}(x), \quad b_{\nu}^{2}=a \psi_{\nu}^{2}, \quad \varphi_{\nu}^{2} b_{\nu}^{2}=a \varphi_{\nu}^{2}
$$

and on $\operatorname{supp} \varphi_{\nu}\left(\right.$ where $\left.\psi_{\nu} \equiv 1\right)$, 


$$
\left\{\begin{array}{l}
b_{\nu}^{\prime}=2^{-1} a^{-1 / 2} a^{\prime} \\
b_{\nu}^{\prime \prime}=-2^{-2} a^{-3 / 2} a^{\prime 2}+2^{-1} a^{-1 / 2} a^{\prime \prime} \\
b_{\nu}^{\prime \prime \prime}=3 \times 2^{-3} a^{-5 / 2} a^{\prime 3}-\frac{3}{4} a^{-3 / 2} a^{\prime} a^{\prime \prime}+2^{-1} a^{-1 / 2} a^{\prime \prime \prime} \\
b_{\nu}^{(4)}=-\frac{15}{16} a^{-7 / 2} a^{\prime^{4}}+\frac{9}{4} a^{-5 / 2} a^{\prime 2} a^{\prime \prime}-\frac{3}{4} a^{-3 / 2} a^{\prime \prime}-a^{-3 / 2} a^{\prime} a^{\prime \prime \prime}+\frac{1}{2} a^{-1 / 2} a^{(4)}
\end{array}\right.
$$

yielding

$$
\left\{\begin{array}{l}
\left|b_{\nu}^{\prime}\right| \leq 2^{-1} a^{-1 / 2}\left|a^{\prime}\right| \lesssim a^{-1 / 2} \rho^{3} \lesssim \rho, \\
\left|b_{\nu}^{\prime \prime}\right| \lesssim a^{-3 / 2} \rho^{6}+a^{-1 / 2} \rho^{2} \lesssim 1 \\
\left|b_{\nu}^{\prime \prime \prime}\right| \lesssim a^{-5 / 2} \rho^{9}+a^{-3 / 2} \rho^{3} \rho^{2}+a^{-1 / 2} \rho \lesssim \rho^{-1}, \\
\left|b_{\nu}^{(4)}\right| \lesssim a^{-7 / 2} \rho^{12}+a^{-5 / 2} \rho^{6} \rho^{2}+a^{-3 / 2} \rho^{4}+a^{-3 / 2} \rho^{3} \rho+a^{-1 / 2} \lesssim \rho^{-2} .
\end{array}\right.
$$

Note in particular that

$$
\left|b_{\nu} b_{\nu}^{(4)}\right|+\left|b_{\nu}^{(1)} b_{\nu}^{(3)}\right|+\left|b_{\nu}^{(2)} b_{\nu}^{(2)}\right| \leq C(\theta) .
$$

The whole difficulty is concentrated on the next case.

The nondegenerate indices $\nu$ are those for which $x_{\nu}$ is $\theta$-nondegenerate. Since $4 r \leq \theta / 8 \leq \theta^{1 / 2}$, we can apply Remark A.1.6 on the product ${ }^{7}$

$$
\begin{aligned}
Q_{\nu}=\left[-\theta^{1 / 4} \rho_{\nu}+x_{\nu 1}, \theta^{1 / 4} \rho_{\nu}+x_{\nu 1}\right] \times B_{\mathbb{R}^{m-1}}\left(x_{\nu}^{\prime}, \theta^{1 / 2} \rho_{\nu}\right) & \\
& \left(\text { here } x_{\nu}=\left(x_{\nu 1}, x_{\nu}^{\prime}\right) \in \mathbb{R} \times \mathbb{R}^{m-1}\right) .
\end{aligned}
$$

There exists $\alpha: B_{\mathbb{R}^{m-1}}\left(x_{\nu}^{\prime}, \theta^{1 / 2} \rho_{\nu}\right) \rightarrow\left[x_{\nu 1}-\theta^{1 / 4} \rho_{\nu}, x_{\nu 1}+\theta^{1 / 4} \rho_{\nu}\right]$ such that

$$
\partial_{1} a\left(\alpha\left(x^{\prime}\right), x^{\prime}\right)=0
$$

and $\partial_{1}^{2} a(x) \geq \rho_{\nu}^{2} / 2$ for $\left|x-x_{\nu}\right| \leq R_{0} \rho_{\nu}$ where $R_{0}=10^{-2}$ according to Lemma A.1.4. We have on $Q_{\nu}$

$$
\begin{aligned}
a(x) & =a\left(x_{1}, x^{\prime}\right) \\
& =\int_{0}^{1}(1-t) \partial_{1}^{2} a\left(\alpha\left(x^{\prime}\right)+t\left(x_{1}-\alpha\left(x^{\prime}\right)\right), x^{\prime}\right) d t\left(x_{1}-\alpha\left(x^{\prime}\right)\right)^{2}+a\left(\alpha\left(x^{\prime}\right), x^{\prime}\right) .
\end{aligned}
$$

According to Remark A.1.6, we recall that we have for $\left|x^{\prime}-x_{\nu}^{\prime}\right| \leq \theta^{1 / 2} \rho_{\nu}$,

$$
\left\{\begin{array}{l}
\left|\alpha\left(x^{\prime}\right)-x_{\nu 1}\right| \leq \theta^{1 / 4} \rho_{\nu} \\
\left|\alpha^{\prime}\left(x^{\prime}\right)\right| \leq 2 \rho_{\nu}^{-2} \rho\left(\alpha\left(x^{\prime}\right), x^{\prime}\right)^{2} \leq 2 C_{0}^{2}=C_{1} \\
\left|\alpha^{\prime \prime}\left(x^{\prime}\right)\right| \leq 2 \rho_{\nu}^{-2}\left(4^{2} C_{0}^{4}+4^{2} C_{0}^{2}+12\right) \rho\left(\alpha\left(x^{\prime}\right), x^{\prime}\right) \leq C_{2} \rho_{\nu}^{-1} \\
\left|\alpha^{\prime \prime \prime}\left(x^{\prime}\right)\right| \leq C_{3} \rho_{\nu}^{-2}
\end{array}\right.
$$

${ }^{7}$ Naturally the choice of the linear coordinates depends on the index $\nu$, according to Remark A.1.6. Note also that $U_{\nu}^{* *} \subset Q_{\nu} \subset B\left(x_{\nu}, R_{0} \rho_{\nu}\right)$ since $4 r \leq \theta^{1 / 2} \leq \theta^{1 / 4} \leq R_{0}$, according to the previous requirements on $r$ and $\theta$ and also to the condition on $\theta$ in Lemma A.1.5. 
with universal constants $C_{j}$. Let us now compute the derivatives of the function

$$
B^{\prime}=B_{\mathbb{R}^{m-1}}\left(x_{\nu}^{\prime}, \theta^{1 / 2} \rho_{\nu}\right) \ni x^{\prime} \mapsto a\left(\alpha\left(x^{\prime}\right), x^{\prime}\right)=c\left(x^{\prime}\right) .
$$

We have, denoting by $\partial_{2}$ the partial derivative with respect to $x^{\prime}$,

$$
\begin{aligned}
c^{\prime}= & \alpha^{\prime} \partial_{1} a+\partial_{2} a=\partial_{2} a \quad\left(\text { here we use the identity } \partial_{\mathbf{1}} \boldsymbol{a}\left(\boldsymbol{\alpha}\left(\boldsymbol{x}^{\prime}\right), \boldsymbol{x}^{\prime}\right) \equiv \mathbf{0}\right), \\
c^{\prime \prime}= & \alpha^{\prime} \partial_{1} \partial_{2} a+\partial_{2}^{2} a, \\
c^{\prime \prime \prime}= & \alpha^{\prime \prime} \partial_{1} \partial_{2} a+\alpha^{\prime 2} \partial_{1}^{2} \partial_{2} a+2 \alpha^{\prime} \partial_{1} \partial_{2}^{2} a+\partial_{2}^{3} a \\
c^{\prime \prime \prime \prime}= & \alpha^{\prime \prime \prime} \partial_{1} \partial_{2} a+3 \alpha^{\prime \prime} \alpha^{\prime} \partial_{1}^{2} \partial_{2} a+3 \alpha^{\prime \prime} \partial_{1} \partial_{2}^{2} a+\alpha^{\prime 3} \partial_{1}^{3} \partial_{2} a \\
& +3 \alpha^{\prime 2} \partial_{1}^{2} \partial_{2}^{2} a+3 \alpha^{\prime} \partial_{1} \partial_{2}^{3} a+\partial_{2}^{4} a
\end{aligned}
$$

and we obtain

$$
\left|c^{\prime}\right| \lesssim \rho^{3}, \quad\left|c^{\prime \prime}\right| \lesssim \rho^{2}, \quad\left|c^{\prime \prime \prime}\right| \lesssim \rho^{-1} \rho^{2}+\rho \sim \rho, \quad\left|c^{\prime \prime \prime \prime}\right| \lesssim \rho^{-2} \rho^{2}+\rho^{-1} \rho+1 \sim 1
$$

so that

$$
c \in C^{3,1}\left(B^{\prime}\right), \quad\left|c^{(j)}\right| \lesssim \rho_{\nu}^{4-j}, \quad 0 \leq j \leq 4
$$

Since $\partial_{1}^{2} a \gtrsim \rho^{2}$ on $Q_{\nu}$, we can define

$$
R(x)=\omega(x)^{1 / 2}, \quad \omega(x)=\int_{0}^{1}(1-t) \partial_{1}^{2} a\left(\alpha\left(x^{\prime}\right)+t\left(x_{1}-\alpha\left(x^{\prime}\right)\right), x^{\prime}\right) d t
$$

Note also that the identity (on $\left.Q_{\nu}\right), a=R(x)^{2}\left(x_{1}-\alpha\right)^{2}+a\left(\alpha\left(x^{\prime}\right), x^{\prime}\right)$ forces the function

$$
B(x)=R(x)^{2}\left(x_{1}-\alpha\right)^{2}
$$

to be $C^{3,1}\left(Q_{\nu}\right)$ with a $j$-th derivative bounded above in absolute value by $\rho_{\nu}^{4-j}$ $(0 \leq j \leq 4)$ since it is the case for $a$ and $c$ (this fact is not obvious since the function $R$ is a priori only $\left.C^{1,1}\right)$. Defining on $Q_{\nu}$

$$
b(x)=R(x)\left(x_{1}-\alpha\left(x^{\prime}\right)\right)
$$

we see that

$$
a=b^{2}+c, \quad\left|\left(b^{2}\right)^{(j)}\right|=\left|B^{(j)}\right| \lesssim \rho_{\nu}^{4-j}, \quad 0 \leq j \leq 4 .
$$

As a consequence with $\beta=x_{1}-\alpha\left(x^{\prime}\right), b^{2}=R^{2} \beta^{2}=B \in C^{3,1}$,

$$
\begin{array}{r}
R^{2} \beta^{2}=\overbrace{B\left(\alpha\left(x^{\prime}\right), x^{\prime}\right)}^{=0}+\overbrace{\int_{0}^{1} \partial_{1} B\left(\alpha\left(x^{\prime}\right)+\theta\left(x_{1}-\alpha\left(x^{\prime}\right)\right), x^{\prime}\right) d \theta \beta,}^{\in C^{2,1}}, \\
\left|\beta^{(j)}\right| \lesssim \rho^{1-j}, \quad 0 \leq j \leq 3,
\end{array}
$$


and since $\beta$ vanishes on an hypersurface

$$
\left\{\begin{array}{l}
R^{2} \beta=\int_{0}^{1} \partial_{1} B\left(\alpha\left(x^{\prime}\right)+\theta\left(x_{1}-\alpha\left(x^{\prime}\right)\right), x^{\prime}\right) d \theta \in C^{2,1} \\
\left|\left(R^{2} \beta\right)^{(j)}\right| \lesssim \rho_{\nu}^{3-j}, 0 \leq j \leq 3, \quad(\text { from }(3.1 .16))
\end{array}\right.
$$

Also we have $0<R^{2}=\omega \in C^{1,1}, \omega \sim \rho_{\nu}^{2}$ and from (3.1.14-11),

$$
\left|\omega^{(j)}\right| \lesssim \rho_{\nu}^{2-j}, \quad 0 \leq j \leq 2
$$

entailing that with $R=\omega^{1 / 2}$,

$$
\begin{aligned}
& \left|R^{\prime}=\frac{1}{2} \omega^{-1 / 2} \omega^{\prime}\right| \lesssim 1 \\
& \left|R^{\prime \prime}=-\frac{1}{4} \omega^{-3 / 2} \omega^{\prime 2}+\frac{1}{2} \omega^{-1 / 2} \omega^{\prime \prime}\right| \lesssim \rho_{\nu}^{-3} \rho_{\nu}^{2}+\rho_{\nu}^{-1} \sim \rho_{\nu}^{-1} .
\end{aligned}
$$

Using Leibniz' formula, we get $\left(R^{2} \beta\right)^{\prime \prime \prime}=(\omega \beta)^{\prime \prime \prime}=\omega^{\prime \prime \prime} \beta+3 \omega^{\prime \prime} \beta^{\prime}+3 \omega^{\prime} \beta^{\prime \prime}+\omega \beta^{\prime \prime \prime}$, which makes sense since $\omega^{\prime \prime \prime}$ is a distribution of order 1 and $\beta$ is $C^{2,1}$ (see (3.1.11)). From (3.1.17), we know that $(\omega \beta)^{\prime \prime \prime}$ is $L^{\infty}$, and since it is also the case of $\omega^{\prime \prime} \beta^{\prime}, \omega^{\prime} \beta^{\prime \prime}, \omega \beta^{\prime \prime \prime}$ from (3.1.18) and (3.1.11), we get that $\omega^{\prime \prime \prime} \beta$ belongs to $L^{\infty}$ and

$$
\left|\omega^{\prime \prime \prime} \beta\right| \lesssim 1
$$

On the other hand we have

$\omega^{\prime \prime \prime}=2\left(R R^{\prime}\right)^{\prime \prime}=2\left(R^{\prime 2}+R R^{\prime \prime}\right)^{\prime}=4 R^{\prime} R^{\prime \prime}+2\left(R R^{\prime \prime}\right)^{\prime}=6 R^{\prime} R^{\prime \prime}+2 \underbrace{R}_{C^{1,1}} \underbrace{R^{\prime \prime \prime}}_{\begin{array}{c}\text { distribution } \\ \text { of order } 1\end{array}}$

entailing from (3.1.20), that $\beta\left(6 R^{\prime} R^{\prime \prime}+2 R R^{\prime \prime \prime}\right)$ is $L^{\infty}$ and since it is the case of $\beta R^{\prime} R^{\prime \prime}$ (from (3.1.11) and (3.1.19)), we get that $\beta R R^{\prime \prime \prime}$ is $L^{\infty}$ and, using Remark 3.1.2, we obtain

(3.1.21) $\left|\beta R R^{\prime \prime \prime}\right| \lesssim 1$, i.e. for all multi-indices $\gamma$ with length $3,\left|\beta R \partial_{x}^{\gamma} R\right| \lesssim 1$.

With $b=R \beta$, we get $b^{\prime} b^{\prime \prime}=\left(R^{\prime} \beta+R \beta^{\prime}\right)\left(R^{\prime \prime} \beta+2 R^{\prime} \beta^{\prime}+R \beta^{\prime \prime}\right)$ and to check that $\left(b^{\prime} b^{\prime \prime}\right)^{\prime}$ is in $L^{\infty}$ with

$$
\left|\left(b^{\prime} b^{\prime \prime}\right)^{\prime}\right| \lesssim 1
$$

it is enough (see (3.1.11) and (3.1.19)) to check the derivatives of $R^{\prime \prime} \beta R^{\prime} \beta$, $R^{\prime \prime} \beta R \beta^{\prime}$ which are, up to bounded terms (see our Appendix A.3 for the meaning of the products)

$$
R^{\prime \prime \prime} \beta R^{\prime} \beta=R^{\prime \prime \prime} \beta R R^{\prime} \frac{\beta}{R}, \quad R^{\prime \prime \prime} \beta R \beta^{\prime}
$$


which are bounded according to 3.1.21-19-11. Note that $b^{\prime \prime}$ is bounded from (3.1.19) and (3.1.11). We want also to verify that $\left(b b^{\prime \prime}\right)^{\prime \prime}$ is bounded. We use that $\left(b^{2}\right)^{(4)}$ is bounded from (3.1.16) and since we have

$$
\underbrace{\left(b^{2}\right)^{\prime \prime \prime \prime \prime}}_{\substack{\text { bounded } \\(3.1 .16)}}=2\left(b^{\prime} \otimes b^{\prime}+b b^{\prime \prime}\right)^{\prime \prime}=2 \underbrace{\left(b^{\prime} \otimes b^{\prime \prime}+b^{\prime \prime} \otimes b^{\prime}\right)^{\prime}}_{\substack{\text { bounded } \\(3.1 .22)}}+2\left(b b^{\prime \prime}\right)^{\prime \prime},
$$

we obtain ${ }^{8}$ the boundedness of $\left(b b^{\prime \prime}\right)^{\prime \prime}$.

Remark 3.1.5. Before going on, we should note that our functions $b, c$ above are only defined on $Q_{\nu}$ where holds the identity $a(x)=b(x)^{2}+c\left(x^{\prime}\right)$. We can replace the function $c$ above by

$$
\tilde{c}\left(x^{\prime}\right)=c\left(x^{\prime}\right) \chi\left(\left(x^{\prime}-x_{\nu}^{\prime}\right) \theta^{-1 / 2} \rho_{\nu}^{-1}\right)
$$

where $\chi \in C_{\mathrm{c}}^{\infty}\left(\mathbb{R}^{m-1}\right)$ supported in the unit ball and equal to 1 in the ball of radius $1 / 2$, so that $\tilde{c}$ is defined on $\mathbb{R}^{m-1}$ and the identity $a=b^{2}+\tilde{c}$ holds on

$$
x_{\nu}+\frac{1}{2}\left(Q_{\nu}-x_{\nu}\right) \supset U_{\nu}^{*} \supset \operatorname{supp} \varphi_{\nu}
$$

The bounds on the derivatives are unchanged as long as $\theta$ is fixed, which is the case.

Taking that remark into account, as well as the above estimates on the derivatives, we have finally, with $E_{2}$ standing for the nondegenerate indices,

$$
\begin{aligned}
a(x)= & \sum_{\nu \in \mathbb{N}} b_{\nu}(x)^{2} \varphi_{\nu}^{2}(x)+\sum_{\nu \in E_{2}} a_{\nu}\left(x^{\prime}\right) \varphi_{\nu}^{2}(x) \\
& \left|b_{\nu}\right| \lesssim \rho_{\nu}^{2},\left|b_{\nu}^{\prime}\right| \lesssim \rho_{\nu},\left|b_{\nu}^{\prime \prime}\right| \lesssim 1,\left|\left(b_{\nu} b_{\nu}^{\prime \prime}\right)^{\prime \prime}\right|+\left|\left(b_{\nu}^{\prime} b_{\nu}^{\prime \prime}\right)^{\prime}\right| \lesssim 1 \\
& \left|a_{\nu}\right| \lesssim \rho_{\nu}^{4},\left|a_{\nu}^{\prime}\right| \lesssim \rho_{\nu}^{3},\left|a_{\nu}^{\prime \prime}\right| \lesssim \rho_{\nu}^{2},\left|a_{\nu}^{\prime \prime \prime}\right| \lesssim \rho_{\nu},\left|a_{\nu}^{\prime \prime \prime \prime}\right| \lesssim 1, \\
& a_{\nu} \text { is defined on } \mathbb{R}^{m-1} .
\end{aligned}
$$

Now, we consider the function $\mathbb{R}^{m-1} \ni t \mapsto A(t)=\rho_{\nu}^{-4} a_{\nu}\left(\rho_{\nu} t\right)$ and we have

$$
\left|A_{\nu}\right| \lesssim 1,\left|A_{\nu}^{\prime}\right| \lesssim 1,\left|A_{\nu}^{\prime \prime}\right| \lesssim 1,\left|A_{\nu}^{\prime \prime \prime}\right| \lesssim 1,\left|A_{\nu}^{\prime \prime \prime \prime}\right| \lesssim 1
$$

\footnotetext{
${ }^{8}$ The equality (3.1.23) is an equality between tensors $(0,4)$ and it might look somewhat pedantic to resort to such notations: the reader may check directly the implication

$$
\begin{gathered}
\left.\begin{array}{r}
\forall \gamma,|\gamma|=4, \partial_{x}^{\gamma}\left(b^{2}\right) \in L^{\infty} \\
\forall \gamma_{j}, 1 \leq j \leq 3,\left|\gamma_{1}\right|=1=\left|\gamma_{2}\right|,\left|\gamma_{3}\right|=2, \partial_{x}^{\gamma_{1}}\left(\partial_{x}^{\gamma_{2}} b \partial_{x}^{\gamma_{3}} b\right) \in L^{\infty},
\end{array}\right\} \\
\Longrightarrow \forall \gamma_{3}, \gamma_{4},\left|\gamma_{3}\right|=2=\left|\gamma_{4}\right|, \partial_{x}^{\gamma_{3}}\left(b \partial_{x}^{\gamma_{4}} b\right) \in L^{\infty}
\end{gathered}
$$
}


Following the main argument in the proof by C. Fefferman and D. H. Phong, we can use an induction on the dimension $m$ to get

$$
A(t)=\sum_{1 \leq j \leq N_{m-1}} B_{j}^{2}(t), \quad B_{j} \in C^{1,1}, \quad \text { and } B_{j}^{\prime \prime},\left(B_{j}^{\prime} B_{j}^{\prime \prime}\right)^{\prime},\left(B_{j} B_{j}^{\prime \prime}\right)^{\prime \prime} \in L^{\infty} .
$$

Incorporated in the induction hypothesis is that the bounds on $B$ depend only on the bounds on $A^{(4)}$. We obtain

$$
a(x)=\sum_{\nu \in \mathbb{N}} b_{\nu}(x)^{2} \varphi_{\nu}^{2}(x)+\sum_{\nu \in E_{2}} \sum_{1 \leq j \leq N_{m-1}} \rho_{\nu}^{4} B_{j, \nu}^{2}\left(\frac{x^{\prime}}{\rho_{\nu}}\right) \varphi_{\nu}^{2}(x)
$$

i.e.

$$
a(x)=\sum_{1 \leq j \leq N_{m-1}+1} \sum_{\nu \in \mathbb{N}} b_{\nu, j}(x)^{2} \varphi_{\nu}^{2}(x) .
$$

One needs to pass to a finite sum, which is quite standard since the overlap of the support of the functions $\varphi_{\nu}$ is bounded; this last argument is given in Appendix A.5. The proof of Theorem 3.1.1 is complete.

\section{§3.2. Application of the Wick calculus: proof of Theorem 1.3.1}

Let $a$ be a nonnegative function defined on $\mathbb{R}^{2 n}$ such that $a^{(4)}$ belongs to $\mathcal{A}$ (defined in Proposition 1.2.1). Applying Lemma 2.2.1 and the $L^{2}$-boundedness of the operators with Weyl symbol in $\mathcal{A}$, we see that it suffices to prove that the operator with Wick symbol $a-\frac{1}{8 \pi}$ trace $a^{\prime \prime}$ is semi-bounded from below. Since $\mathcal{A} \subset L^{\infty}\left(\mathbb{R}^{2 n}\right)$, it is enough to prove the following lemma.

Lemma 3.2.1. Let a be a nonnegative function defined on $\mathbb{R}^{2 n}$ such that $a^{(4)}$ belongs to $L^{\infty}\left(\mathbb{R}^{2 n}\right)$. Theorem 3.1 .1 is providing a decomposition $a=$ $\sum_{1 \leq j \leq N} b_{j}^{2}$ along with the estimates (3.1.2). Then we have

$$
\left(a-\frac{1}{8 \pi} \operatorname{trace} a^{\prime \prime}\right)^{\text {Wick }}=\sum_{1 \leq j \leq N}\left[\left(b_{j}-\frac{1}{8 \pi} \operatorname{trace} b_{j}^{\prime \prime}\right)^{\text {Wick }}\right]^{2}+R
$$

where $R$ is a $L^{2}$-bounded operator such that $\|R\|_{\mathcal{L}\left(L^{2}\left(\mathbb{R}^{n}\right)\right)} \leq C\left\|a^{(4)}\right\|_{L^{\infty}\left(\mathbb{R}^{2 n}\right)}, C$ depending only on the dimension $n$.

Proof. We have

$$
a-\frac{1}{8 \pi} \operatorname{trace} a^{\prime \prime}=a-\frac{\Delta a}{8 \pi}=\sum_{1 \leq j \leq N} b_{j}^{2}-\frac{1}{4 \pi}\left|\nabla b_{j}\right|^{2}-\frac{1}{4 \pi} b_{j} \Delta b_{j} .
$$


Then using Lemma 2.3.3, we get

$$
b_{j}^{\mathrm{Wick}} b_{j}^{\mathrm{Wick}}=\left(b_{j}^{2}-\frac{1}{4 \pi}\left|\nabla b_{j}\right|^{2}\right)^{\mathrm{Wick}}+S_{j},
$$

with

$$
\begin{aligned}
\left\|S_{j}\right\|_{\mathcal{L}\left(L^{2}\left(\mathbb{R}^{n}\right)\right)} & \leq C_{1}\left(\left\|b_{j}^{\prime \prime}\right\|_{L^{\infty}}^{2}+\left\|\left(b_{j}^{\prime \prime} b_{j}^{\prime}\right)^{\prime}\right\|_{L^{\infty}}+\left\|\left(b_{j} b_{j}^{\prime \prime}\right)^{\prime \prime}\right\|_{L^{\infty}}\right) \\
& \leq C_{2}\left\|a^{(4)}\right\|_{L^{\infty}\left(\mathbb{R}^{2 n}\right)},
\end{aligned}
$$

where $C_{1}, C_{2}$ depend only on the dimension. Moreover, we have, from Lemma 2.3.1,

$$
\operatorname{Re}\left(b_{j}^{\mathrm{Wick}}\left(\Delta b_{j}\right)^{\mathrm{Wick}}\right)=\left(b_{j} \Delta b_{j}-\frac{1}{4 \pi} \nabla \cdot\left(\nabla b_{j} \Delta b_{j}\right)+\frac{1}{4 \pi}\left(\Delta b_{j}\right)^{2}\right)^{\mathrm{Wick}}+R_{j},
$$

with

$$
\left\|R_{j}\right\|_{\mathcal{L}\left(L^{2}\left(\mathbb{R}^{n}\right)\right)} \leq C_{3}\left\|b_{j}^{\prime \prime}\right\|_{L^{\infty}\left(\mathbb{R}^{2 n}\right)}^{2} \leq C_{4}\left\|a^{(4)}\right\|_{L^{\infty}\left(\mathbb{R}^{2 n}\right)} .
$$

As a consequence, from $(3.2 .2-3)$, we get

$$
\begin{aligned}
& \left(b_{j}-\frac{1}{8 \pi} \operatorname{trace} b_{j}^{\prime \prime}\right)^{\mathrm{Wick}}\left(b_{j}-\frac{1}{8 \pi} \operatorname{trace} b_{j}^{\prime \prime}\right)^{\mathrm{Wick}} \\
= & \left(b_{j}^{2}-\frac{1}{4 \pi}\left|\nabla b_{j}\right|^{2}-\frac{1}{4 \pi} b_{j} \Delta b_{j}\right)^{\mathrm{Wick}}+\frac{1}{16 \pi^{2}}\left(\nabla \cdot\left(\nabla b_{j} \Delta b_{j}\right)\right)^{\mathrm{Wick}} \\
& \quad-\frac{1}{16 \pi^{2}}\left(\left(\Delta b_{j}\right)^{2}\right)^{\mathrm{Wick}}+S_{j}-\frac{1}{4 \pi} R_{j}+\frac{1}{64 \pi^{2}}\left(\Delta b_{j}\right)^{\mathrm{Wick}}\left(\Delta b_{j}\right)^{\mathrm{Wick}}
\end{aligned}
$$

so that from $(2.1 .2),(3.1 .2)$ and the estimates above for $R_{j}, S_{j}$, we obtain from (3.2.1) that

$$
\begin{gathered}
\sum_{1 \leq j \leq N}\left(b_{j}-\frac{1}{8 \pi} \operatorname{trace} b_{j}^{\prime \prime}\right)^{\text {Wick }}\left(b_{j}-\frac{1}{8 \pi} \operatorname{trace} b_{j}^{\prime \prime}\right)^{\text {Wick }} \\
=\left(a-\frac{1}{8 \pi} \operatorname{trace} a^{\prime \prime}\right)^{\text {Wick }}+S
\end{gathered}
$$

with $\|S\|_{\mathcal{L}\left(L^{2}\left(\mathbb{R}^{n}\right)\right)} \leq C_{5}\left\|a^{(4)}\right\|_{L^{\infty}\left(\mathbb{R}^{2 n}\right)} C_{5}$ depending only on the dimension. This is the result of the lemma, completing as well the proof of Theorem 1.3.1.

Remark. The proof above is giving a slightly better result, since we prove the lemma for each $a_{j}=b_{j}^{2}$, provided the lhs of (3.1.2) is controlled.

Comment 3.2.2. One may ask the following question: why did we not apply this induction argument on the Sjöstrand algebra $\mathcal{A}$ directly, and avoid that 
complicated detour with the Wick calculus? The answer to that interrogation is simple: as seen above the Fefferman-Phong induction procedure requires a cutting process (this is the metric $\left.d X^{2} / \rho(X)^{2}\right)$ and also a bending of the phase space (the function $\alpha$ is not linear). Although the cutting part may respect $\mathcal{A}$, it is not very likely that the rigid affine structure of $\mathcal{A}$ would survive the bending.

\section{$\S 3.3 . \quad$ Proof of Corollary 1.3.2}

Let us begin with the statement (iv) in this corollary. Let us define

$$
A(x, \xi)=h^{-2} a\left(x h^{1 / 2}, \xi h^{-1 / 2}, h\right) .
$$

The function $A$ satisfies

$$
\begin{aligned}
\left(\partial_{\xi}^{\alpha} \partial_{x}^{\beta} A\right)(x, \xi) & =h^{-2-\frac{|\alpha|}{2}+\frac{|\beta|}{2}}\left(\partial_{1}^{\beta} \partial_{2}^{\alpha} a\right)\left(x h^{1 / 2}, \xi h^{-1 / 2}, h\right) \\
& =h^{\frac{|\alpha|+|\beta|-4}{2}}\left(\partial_{1}^{\beta} \partial_{2}^{\alpha} a\right)\left(x h^{1 / 2}, \xi h^{-1 / 2}, h\right) h^{-|\alpha|}
\end{aligned}
$$

so that for $|\alpha|+|\beta|=4$, we have $\left(\partial_{\xi}^{\alpha} \partial_{x}^{\beta} A\right)(x, \xi)=\left(\partial_{1}^{\beta} \partial_{2}^{\alpha} a\right)\left(x h^{1 / 2}, \xi h^{-1 / 2}\right.$, $h) h^{-|\alpha|}$. We have supposed that for $|\alpha|+|\beta|=4$, the functions $(x, \xi) \mapsto$ $\left(\partial_{1}^{\beta} \partial_{2}^{\alpha} a\right)\left(x h^{1 / 2}, \xi h^{-1 / 2}, h\right) h^{-|\alpha|}$ belongs to $\mathcal{A}$ with a norm bounded above independently by $\nu_{0}$. As a result the function $A^{(4)}(x, \xi)$ belongs to $\mathcal{A}$ with a norm bounded above by $\nu_{0}$. Since $A(x, \xi) \geq 0$, Theorem 1.3.1 implies that $A^{w}+C_{n} \nu_{0} \geq 0$, i.e. $\left(a\left(x h^{1 / 2}, \xi h^{-1 / 2}, h\right)\right)^{w}+C_{n} \nu_{0} h^{2} \geq 0$ and since there is a unitary mapping $U_{h}$ such that $U_{h}^{*} a(x, \xi, h)^{w} U_{h}=\left(a\left(x h^{1 / 2}, \xi h^{-1 / 2}, h\right)\right)^{w}$, we obtain

$$
a(x, \xi, h)^{w}+C_{n} \nu_{0} h^{2} \geq 0, \text { qed }
$$

To get that Re $a(x, D, h)+C h^{2} \geq 0$, one ${ }^{9}$ should note that the symbols $A^{(4)}$ defined above belong to $\mathcal{A}$, which implies that it is also the case for $J^{-1 / 2} A^{(4)}$ and $J^{1 / 2} \overline{A^{(4)}}$. Now we have

$$
2 \operatorname{Re} a(x, D, h)=2 \operatorname{Re}\left(J^{-1 / 2} a\right)^{w}=\left(J^{-1 / 2} a+J^{1 / 2} \bar{a}\right)^{w},
$$

so that rescaling ${ }^{10}$ the symbol $J^{-1 / 2} a+J^{1 / 2} \bar{a}$, we find $J^{-1 / 2} A+J^{1 / 2} \bar{A}$. Since

\footnotetext{
${ }^{9}$ With the group $J^{t}$ defined in Proposition 1.2.3, the formula linking the Weyl quantization with the ordinary quantization is $a(x, D)=\left(J^{-1 / 2} a\right)^{w}$.

${ }^{10}$ We define

$$
\begin{aligned}
B(x, \xi) & =h^{-2}\left(J^{-1 / 2} a\right)\left(x h^{1 / 2}, \xi h^{-1 / 2}\right)+h^{-2}\left(J^{1 / 2} a\right)\left(x h^{1 / 2}, \xi h^{-1 / 2}\right) \\
& =\left(J^{-1 / 2} A\right)(x, \xi)+\left(J^{1 / 2} A\right)(x, \xi) .
\end{aligned}
$$
}


we have

$J^{-1 / 2} A=e^{-i \pi D_{x} \cdot D_{\xi}} A=A-i \pi D_{x} \cdot D_{\xi} A-\int_{0}^{1}(1-\theta) e^{-i \pi \theta D_{x} \cdot D_{\xi}} d \theta \pi^{2}\left(D_{x} \cdot D_{\xi}\right)^{2} A$,

and that $A$ is real-valued, we get

$$
\operatorname{Re}\left(J^{-1 / 2} A\right)=A-\int_{0}^{1}(1-\theta) e^{-i \pi \theta D_{x} \cdot D_{\xi}} d \theta \pi^{2} \underbrace{\left(D_{x} \cdot D_{\xi}\right)^{2} A}_{\in \mathcal{A}} .
$$

Now we have from the previous identity, since $\mathcal{A}$ is stable by the group $J^{t}$ (Theorem 1.1 in [S1]), with a uniform constant for $t$ in a compact set,

$$
2 \operatorname{Re} A(x, D)=\left(2 \operatorname{Re}\left(J^{-1 / 2} A\right)\right)^{w} \in 2 A^{w}+\mathcal{A}^{w} .
$$

We can then apply the result (3.3.2) and the $L^{2}$ boundedness of $\mathcal{A}^{w}$ to conclude. The proof of (iv) in Corollary 1.3.2 is complete.

Let us show that (iv) implies (iii). We define $b(x, \xi, h)=a(x, h \xi)$, which is nonnegative; it is enough to check the functions $(x, \xi) \mapsto\left(\partial_{1}^{\beta} \partial_{2}^{\alpha} b\right)\left(x h^{1 / 2}, \xi h^{-1 / 2}\right.$, $h) h^{-|\alpha|}$, for $|\alpha|+|\beta|=4$. We have in fact

$$
\left(\partial_{1}^{\beta} \partial_{2}^{\alpha} b\right)\left(x h^{1 / 2}, \xi h^{-1 / 2}, h\right) h^{-|\alpha|}=\left(\partial_{1}^{\beta} \partial_{2}^{\alpha} a\right)\left(x h^{1 / 2}, \xi h^{1 / 2}\right) .
$$

Now, from Lemma A.2.1 in our appendix, for $h \in(0,1]$, the functions

$$
(x, \xi) \mapsto\left(\partial_{1}^{\beta} \partial_{2}^{\alpha} a\right)\left(x h^{1 / 2}, \xi h^{1 / 2}\right)
$$

belong to $\mathcal{A}$ with a bounded norm since we have supposed that $a^{(4)} \in \mathcal{A}$. We can then apply the already proven result (iv) in the corollary to get

$$
a(x, \xi h)^{w}+C h^{2}\left\|a^{(4)}\right\|_{\mathcal{A}} \geq 0, \quad \operatorname{Re} a(x, h D)+C h^{2}\left\|a^{(4)}\right\|_{\mathcal{A}} \geq 0, \quad \text { qed. }
$$

Let us show that (iv) implies (ii). We assume that $a(x, \xi, h)$ is a nonnegative function satisfying the assumptions of (ii). According to the already proven (iv), we need only to check, for $\left|\alpha^{\prime}\right|+\left|\beta^{\prime}\right|=4$, the norm in $\mathcal{A}$ of

$$
(x, \xi) \mapsto\left(\partial_{1}^{\beta^{\prime}} \partial_{2}^{\alpha^{\prime}} a\right)\left(x h^{1 / 2}, \xi h^{-1 / 2}, h\right) h^{-\left|\alpha^{\prime}\right|}=c_{\alpha^{\prime} \beta^{\prime}}(x, \xi) .
$$

Because of the second inclusion in (1.2.1), it is enough to find an $L^{\infty}$ bound on the $2 n+1$ first derivatives of that function; we have, for $\left|\alpha^{\prime \prime}\right|+\left|\beta^{\prime \prime}\right| \leq 2 n+1$

$$
\left(\partial_{\xi}^{\alpha^{\prime \prime}} \partial_{x}^{\beta^{\prime \prime}} c_{\alpha^{\prime} \beta^{\prime}}\right)(x, \xi)=\left(\partial_{1}^{\beta^{\prime}+\beta^{\prime \prime}} \partial_{2}^{\alpha^{\prime}+\alpha^{\prime \prime}} a\right)\left(x h^{1 / 2}, \xi h^{-1 / 2}, h\right) h^{-\left|\alpha^{\prime}\right|} h^{\frac{-\left|\alpha^{\prime \prime}\right|+\left|\beta^{\prime \prime}\right|}{2}}
$$


and from the assumption in (ii), we get, since $4 \leq\left|\alpha^{\prime}+\alpha^{\prime \prime}\right|+\left|\beta^{\prime}+\beta^{\prime \prime}\right| \leq 2 n+5$,

$$
\left|\left(\partial_{1}^{\beta^{\prime}+\beta^{\prime \prime}} \partial_{2}^{\alpha^{\prime}+\alpha^{\prime \prime}} a\right)\left(x h^{1 / 2}, \xi h^{-1 / 2}, h\right)\right| \leq C_{\alpha^{\prime}+\alpha^{\prime \prime}, \beta^{\prime}+\beta^{\prime \prime}} h^{\left|\alpha^{\prime}\right|+\left|\alpha^{\prime \prime}\right|},
$$

so that (3.3.3-4) imply

$$
\begin{aligned}
\left|\left(\partial_{\xi}^{\alpha^{\prime \prime}} \partial_{x}^{\beta^{\prime \prime}} c_{\alpha^{\prime} \beta^{\prime}}\right)(x, \xi)\right| & \leq C_{\alpha^{\prime}+\alpha^{\prime \prime}, \beta^{\prime}+\beta^{\prime \prime}} h^{\left|\alpha^{\prime}\right|+\left|\alpha^{\prime \prime}\right|} h^{-\left|\alpha^{\prime}\right|} h^{\frac{-\left|\alpha^{\prime \prime}\right|+\left|\beta^{\prime \prime}\right|}{2}} \\
& =C_{\alpha^{\prime}+\alpha^{\prime \prime}, \beta^{\prime}+\beta^{\prime \prime}} h^{\frac{\left|\alpha^{\prime \prime}\right|+\left|\beta^{\prime \prime}\right|}{2}} \leq C_{\alpha^{\prime}+\alpha^{\prime \prime}, \beta^{\prime}+\beta^{\prime \prime}}
\end{aligned}
$$

yielding the sought bound. The proof of (ii) is complete.

Proof of (i) in Corollary 1.3.2. Using a Littlewood-Paley decomposition, we have

$$
\begin{gathered}
1=\sum_{\nu \geq 0} \varphi_{\nu}^{2}(\xi), \quad \varphi_{\nu} \in C_{\mathrm{c}}^{\infty}\left(\mathbb{R}^{n}\right), \\
\text { for } \nu \geq 1, \operatorname{supp} \varphi_{\nu} \subset\left\{2^{\nu-1} \leq|\xi| \leq 2^{\nu+1}\right\}, \quad \sup _{\nu, \xi}\left|\partial_{\xi}^{\alpha} \varphi_{\nu}(\xi)\right| 2^{\nu|\alpha|}<\infty .
\end{gathered}
$$

We introduce also some smooth nonnegative compactly supported functions $\psi_{\nu}(\xi)$, satisfying the same uniform estimates than $\varphi_{\nu}$ and supported in $2^{\nu-3} \leq$ $|\xi| \leq 2^{\nu+3}$ for $\nu \geq 1$, identically 1 on $2^{\nu-2} \leq|\xi| \leq 2^{\nu+2}$ (in particular on the support of $\varphi_{\nu}$ ). We consider a nonnegative symbol $a$ satisfying (1.1.1) for $4 \leq|\alpha|+|\beta| \leq 2 n+5$. We write

$$
a=\sum_{\nu \geq 0} \varphi_{\nu}^{2} a=\sum_{\nu \geq 0}\left(\psi_{\nu \sharp} \varphi_{\nu}^{2} a \sharp \psi_{\nu}+r_{\nu}\right) .
$$

The proof relies on the following

Claim 3.3.1. The operator with Weyl symbol $\sum_{\nu} r_{\nu}$ is bounded on $L^{2}\left(\mathbb{R}^{n}\right)$.

As a matter of fact, if this claim is proven, we are left with the operator $\sum_{\nu} \psi_{\nu}^{w}\left(\varphi_{\nu}^{2} a\right)^{w} \psi_{\nu}^{w}$ and we can apply the already proven result (ii) in this corollary to get that with a uniform $C$,

$$
\sum_{\nu} \psi_{\nu}^{w}\left(\varphi_{\nu}^{2} a\right)^{w} \psi_{\nu}^{w}=\sum_{\nu} \psi_{\nu}^{w} \underbrace{\left(\left(\varphi_{\nu}^{2} a\right)^{w}+C\right)}_{\geq 0} \psi_{\nu}^{w}-C \underbrace{\left(\sum_{\nu} \psi_{\nu}^{2}\right)^{w}}_{L^{2} \text { bounded }}
$$

and so this operator is semi-bounded from below as well as $a^{w}$. Let us prove the claim. We leave as an exercise for the reader to check, using (1.2.2), the 
composition formula

$$
\begin{aligned}
& \left(a_{1} \sharp a_{2} \sharp a_{3}\right)(X) \\
= & 2^{2 n} \iint_{\mathbb{R}^{2 n} \times \mathbb{R}^{2 n}} a_{1}\left(Y_{1}\right) a_{2}\left(Y_{2}\right) a_{3}\left(X-Y_{1}+Y_{2}\right) e^{-4 i \pi\left[X-Y_{1}, X-Y_{2}\right]} d Y_{1} d Y_{2} .
\end{aligned}
$$

Applying this to $\psi_{\nu} \sharp a_{\nu} \sharp \psi_{\nu}$ with $a_{\nu}=\varphi_{\nu}^{2} a$, we get

$$
\begin{gathered}
r_{\nu}(x, \xi)=-2^{n} \iiint_{0}^{1}(1-\theta) e^{-4 i \pi y \eta} \psi_{\nu}(\xi+\eta) \psi_{\nu}(\xi-\eta) \\
\times\left(\partial_{x}^{2} a_{\nu}\right)(x+\theta y, \xi) y^{2} d y d \eta d \theta \\
=\frac{2^{n}}{16 \pi^{2}} \iiint_{0}^{1}(1-\theta) \partial_{\eta}^{2}\left(e^{-4 i \pi y \eta}\right) \psi_{\nu}(\xi+\eta) \\
\quad \times \psi_{\nu}(\xi-\eta)\left(\partial_{x}^{2} a_{\nu}\right)(x+\theta y, \xi) d y d \eta d \theta \\
=\frac{2^{n}}{16 \pi^{2}} \iiint_{0}^{1}(1-\theta) e^{-4 i \pi y \eta} \partial_{\eta}^{2}\left(\psi_{\nu}(\xi+\eta) \psi_{\nu}(\xi-\eta)\right) \\
\times\left(\partial_{x}^{2} a_{\nu}\right)(x+\theta y, \xi) d y d \eta d \theta .
\end{gathered}
$$

From this formula we see that $r_{\nu}$ is supported where $2^{\nu-1} \leq|\xi| \leq 2^{\nu+1}$ since it is the case for $a_{\nu}(\nu \geq 1)$; since the overlap of the rings where $|\xi| \sim 2^{\nu}$ is bounded, it is enough to check some bounds on the derivatives of $r_{\nu}$ to get similar bounds on the $\sum_{\nu} r_{\nu}$. Moreover in the integrand, if the function $\psi_{\nu}(\xi+\eta)$ is differentiated, we get

$$
2^{\nu+2} \leq|\xi+\eta| \leq 2^{\nu+3} \quad \text { or } \quad 2^{\nu-3} \leq|\xi+\eta| \leq 2^{\nu-2} .
$$

As a result, in the first case, we have $|\eta| \geq|\xi+\eta|-|\xi| \geq 2^{\nu+2}-2^{\nu+1}=2^{\nu+1}$, whereas in the second case $|\eta| \geq|\xi|-|\xi+\eta| \geq 2^{\nu-1}-2^{\nu-2}=2^{\nu-2}$, which implies that we always have $|\eta| \geq 2^{\nu-2}$. Since we have also $|\eta| \leq|\xi+\eta|+|\xi| \leq$ $2^{\nu+3}+2^{\nu+1}$, we obtain (note that the case when the other function $\psi(\xi-\eta)$ is differentiated is similar) on the integrand

$$
2^{\nu-2} \leq|\eta| \leq 2^{\nu+4} .
$$

We write now

$$
\begin{aligned}
& \frac{1}{\alpha !}\left(\partial_{\xi}^{\alpha} \partial_{x}^{\beta} r_{\nu}\right)(x, \xi)=\sum_{\alpha^{\prime}+\alpha^{\prime \prime}=\alpha} \frac{2^{n}}{\alpha^{\prime} ! \alpha^{\prime \prime} ! 16 \pi^{2}} \\
& \times \iiint_{0}^{1}(1-\theta) e^{-4 i \pi y \eta} \partial_{\xi}^{\alpha^{\prime}} \partial_{\eta}^{2}\left(\psi_{\nu}(\xi+\eta) \psi_{\nu}(\xi-\eta)\right)\left(\partial_{\xi}^{\alpha^{\prime \prime}} \partial_{x}^{\beta} \partial_{x}^{2} a_{\nu}\right)(x+\theta y, \xi) d y d \eta d \theta
\end{aligned}
$$


and since the integral above is, for $N, k$ even integers, $N>n$,

$$
\begin{aligned}
& \iiint_{0}^{1}(1-\theta) e^{-4 i \pi y \eta}(1+4 \mid\left.\left.\eta\right|^{2}\right)^{-k / 2} \\
& \times\left(1+4|y|^{2}\right)^{-N / 2}\left(1+D_{\eta}^{2}\right)^{N / 2} \partial_{\xi}^{\alpha^{\prime}} \partial_{\eta}^{2}\left(\psi_{\nu}(\xi+\eta) \psi_{\nu}(\xi-\eta)\right) \\
& \times\left(1+D_{y}^{2}\right)^{k / 2}\left(\left(\partial_{\xi}^{\alpha^{\prime \prime}} \partial_{x}^{\beta} \partial_{x}^{2} a_{\nu}\right)(x+\theta y, \xi)\right) d y d \eta d \theta
\end{aligned}
$$

we get, for $|\alpha|+|\beta|+k \leq 2 n+3$,

$$
\left|\left(\partial_{\xi}^{\alpha} \partial_{x}^{\beta} r_{\nu}\right)(x, \xi)\right| \leq C_{\alpha \beta N}\left(2^{\nu}\right)^{-k-\left|\alpha^{\prime}\right|-2+2-\left|\alpha^{\prime \prime}\right|+n}=C_{\alpha \beta N}\left(2^{\nu}\right)^{-|\alpha|+n-k} .
$$

For $\alpha, \beta$ given such that $\max (|\alpha|,|\beta|) \leq n+1$, we choose $k=n-|\alpha|$ or $k=n-|\alpha|+1$ so that $k$ is even, and we get, uniformly in $\nu,\left|\left(\partial_{\xi}^{\alpha} \partial_{x}^{\beta} r_{\nu}\right)(x, \xi)\right| \lesssim 1$; note that then we have indeed

$$
|\alpha|+|\beta|+k \leq|\beta|+n+1 \leq 2 n+2 \leq 2 n+3 .
$$

Eventually, from (a mild version of) Theorem 1.2 in [B2] we get Claim 3.3.1: we have proven that for $\max (|\alpha|,|\beta|) \leq n+1, \partial_{\xi}^{\alpha} \partial_{x}^{\beta} r$ is bounded. The proof of (1.1.3) is complete, under the assumptions of the corollary.

Proof of (1.1.2). To obtain also the result for the ordinary quantization is not a direct consequence of the previous result, because of our limitation on the regularity of $a$. So we have to revisit our argument above, replacing at each step the Weyl quantization by the standard quantization. It is a bit tedious, but unavoidable. We write

$$
a=\sum_{\nu \geq 0} \varphi_{\nu}^{2} a=\sum_{\nu \geq 0}\left(\psi_{\nu} \circ \varphi_{\nu}^{2} a \circ \psi_{\nu}+s_{\nu}\right) .
$$

The proof relies on the following

Claim 3.3.2. The operator with standard symbol $\sum_{\nu} s_{\nu}$ is bounded on $L^{2}\left(\mathbb{R}^{n}\right)$.

As a matter of fact, if this claim is proven, we are left with the operator

$$
\operatorname{Re} \sum_{\nu} \mathrm{Op}\left(\psi_{\nu}\right) \mathrm{Op}\left(\varphi_{\nu}^{2} a\right) \mathrm{Op}\left(\psi_{\nu}\right)
$$

and we can apply the already proven result (ii) in this corollary to get that 
with a uniform $C$,

$$
\begin{aligned}
& \sum_{\nu} \operatorname{Op}\left(\psi_{\nu}\right) \operatorname{Re} \operatorname{Op}\left(\varphi_{\nu}^{2} a\right) \operatorname{Op}\left(\psi_{\nu}\right) \\
&=\sum_{\nu} \mathrm{Op}\left(\psi_{\nu}\right) \underbrace{\left(\operatorname{ReOp}\left(\varphi_{\nu}^{2} a\right)+C\right)}_{\geq 0} \operatorname{Op}\left(\psi_{\nu}\right)-\underbrace{C \operatorname{Op}\left(\sum_{\nu} \psi_{\nu}^{2}\right)}_{L^{2} \text { bounded }}
\end{aligned}
$$

and so this operator is semi-bounded from below as well as $\operatorname{Re} O p(a)$. Let us prove the claim. Reminding the ordinary composition formula, we have

$$
(a \circ b)(x, \xi)=\iint_{\mathbb{R}^{n} \times \mathbb{R}^{n}} e^{-2 i \pi y \eta} a(x, \xi+\eta) b(y+x, \xi) d y d \eta .
$$

Applying this to $\psi_{\nu} \circ a_{\nu} \circ \psi_{\nu}$ with $a_{\nu}=\varphi_{\nu}^{2} a$, we get $\psi_{\nu} \circ a_{\nu} \circ \psi_{\nu}=\psi_{\nu} \circ a_{\nu} \psi_{\nu}=$ $\psi_{\nu} \circ a_{\nu}$ and

$$
\begin{aligned}
\left(\psi_{\nu} \circ a_{\nu} \circ \psi_{\nu}\right)(x, \xi)= & \iint e^{-2 i \pi y \eta} \psi_{\nu}(\xi+\eta) a_{\nu}(y+x, \xi) d y d \eta \\
= & \iint e^{-2 i \pi y \eta} \psi_{\nu}(\xi+\eta)\left(a_{\nu}(x, \xi)\right. \\
& \left.+\int_{0}^{1}(1-\theta) \partial_{x}^{2} a_{\nu}(x+\theta y, \xi) y^{2} d \theta\right) d y d \eta \\
= & \left(a_{\nu} \psi_{\nu}\right)(x, \xi)-s_{\nu}(x, \xi)
\end{aligned}
$$

with

$$
s_{\nu}(x, \xi)=-\iiint_{0}^{1}(1-\theta) e^{-2 i \pi y \eta} \psi_{\nu}(\xi+\eta)\left(\partial_{x}^{2} a_{\nu}\right)(x+\theta y, \xi) y^{2} d y d \eta d \theta .
$$

That formula is so similar to the defining formula of $r_{\nu}$ above that we can resume the discussion and use (a mild version of) Theorem 1.1 in [B2] we get Claim 3.3.3: The proof of (1.1.2) is complete, under the assumptions of the corollary.

\section{A. Appendix}

\section{A.1. On nonnegative functions}

Let $a$ be a nonnegative $C^{3,1}$ function defined on $\mathbb{R}^{m}$ such that $\left\|a^{(4)}\right\|_{L^{\infty}} \leq$ $1 ; \rho$ and $\Omega$ are defined in (3.1.3).

Lemma A.1.1. Let $a, \rho, \Omega$ be as above. For $0 \leq j \leq 4$, we have $\left\|a^{(j)}(x)\right\| \leq \gamma_{j} \rho(x)^{4-j}$, with $\gamma_{0}=\gamma_{2}=\gamma_{4}=1, \gamma_{1}=3, \gamma_{3}=4$. 
Proof. The inequalities for $j=0,2,4$ are obvious. Let us write Taylor's formula,

$a(x+h)=a(x)+a^{\prime}(x) h+\frac{1}{2} a^{\prime \prime}(x) h^{2}+\frac{1}{6} a^{(3)}(x) h^{3}+\int_{0}^{1} \frac{(1-\theta)^{3}}{3 !} a^{(4)}(x+\theta h) d \theta h^{4}$.

We get $a(x+h)-a(x)-\frac{1}{2} a^{\prime \prime}(x) h^{2}-\frac{|h|^{4}}{24} \leq a^{\prime}(x) h+\frac{1}{6} a^{(3)}(x) h^{3}$ and since $a(x+h) \geq 0$, we have $-a(x)-\frac{1}{2} a^{\prime \prime}(x) h^{2}-\frac{|h|^{4}}{24} \leq a^{\prime}(x) h+\frac{1}{6} a^{(3)}(x) h^{3}$. Since the rhs is odd in the variable $h$, we obtain

$$
\left|a^{\prime}(x) h+\frac{1}{6} a^{(3)}(x) h^{3}\right| \leq a(x)+\frac{1}{2} a^{\prime \prime}(x) h^{2}+\frac{|h|^{4}}{24} .
$$

Let us choose $h=\rho(x) s T$ where $T$ is a unit vector and $s$ is a real parameter. We have

$$
\left|s \rho(x) a^{\prime}(x) T+s^{3} \rho(x)^{3} \frac{1}{6} a^{(3)}(x) T^{3}\right| \leq \rho(x)^{4}\left(1+\frac{1}{2} s^{2}+\frac{s^{4}}{24}\right) .
$$

Note. Let $\alpha, \beta, \gamma \in \mathbb{R}$, and assume that $\forall s \in \mathbb{R},\left|s \alpha+s^{3} \beta\right| \leq \gamma\left(1+\frac{1}{2} s^{2}+\frac{s^{4}}{24}\right)$. Applying that inequality for $s=1,3$ gives $|\alpha+\beta| \leq \gamma \frac{37}{24},|3 \alpha+27 \beta| \leq \gamma \frac{213}{24}$ and thus

$$
\begin{gathered}
24|\beta|=|3 \alpha+27 \beta-3(\alpha+\beta)| \leq \frac{324}{24} \gamma, \quad|\beta| \leq \gamma \frac{324}{24^{2}}, \\
|\alpha|=|\alpha+\beta-\beta| \leq \gamma \frac{37 \times 24+324}{24^{2}}=\gamma \frac{1212}{576} .
\end{gathered}
$$

As a result, from (A.1.2), we get for $\rho(x)>0,\left\|a^{\prime}(x)\right\| \leq 3 \rho(x)^{3},\left\|a^{(3)}(x)\right\| \leq$ $4 \rho(x)$. If $\rho(x)=0$, we use the inequality (A.1.1) with $h=\epsilon T$ where $T$ is a unit vector and $\epsilon$ is a positive parameter, providing $\left|\epsilon a^{\prime}(x) T+\epsilon^{3} \frac{1}{6} a^{(3)}(x) T^{3}\right| \leq \frac{\epsilon^{4}}{24}$. Dividing by $\epsilon$ and letting it go to zero, we find $a^{\prime}(x) T=0$, for all $T$, i.e. $a^{\prime}(x)=0$. Next we find that for all vectors $T, a^{(3)}(x) T^{3}=0$, implying that the symmetric trilinear form $a^{(3)}(x)$ is zero (see Remark 3.1.2). The proof of the lemma is complete.

Lemma A.1.2. Let $a, \rho, \Omega$ be as above. The metric $\frac{|d x|^{2}}{\rho(x)^{2}}$ is slowly varying on the open set $\Omega$, i.e. there exists $C_{0} \geq 1>r_{0}>0$ such that

$$
x \in \Omega \text { and }|x-y| \leq r_{0} \rho(x) \Longrightarrow y \in \Omega, \quad C_{0}^{-1} \leq \frac{\rho(x)}{\rho(y)} \leq C_{0} .
$$

The constants $r_{0}$ and $C_{0}$ can be chosen as "universal" fixed constants (independently of the dimension and of the function a, which is normalized by the condition $\left.\left\|a^{(4)}\right\|_{L^{\infty}} \leq 1\right)$. 
Proof. Using Taylor's formula, one gets, using (A.1.1), Lemma A.1.1 and Remark 3.1.2,

$$
\begin{aligned}
\rho(x+h)^{4}= & a(x+h)+\left\|a^{\prime \prime}(x+h)\right\|^{2} \\
\leq & a(x)+a^{\prime}(x) h+\frac{1}{2} a^{\prime \prime}(x) h^{2}+\frac{1}{6} a^{\prime \prime \prime}(x) h^{3}+\frac{1}{24}|h|^{4} \\
& +3\left\|a^{\prime \prime}(x)\right\|^{2}+3\left\|a^{\prime \prime \prime}(x)\right\|^{2}|h|^{2}+3 \frac{1}{4}|h|^{4} \\
\leq & 2 a(x)+a^{\prime \prime}(x) h^{2}+\frac{1}{12}|h|^{4}+3\left\|a^{\prime \prime}(x)\right\|^{2}+3\left\|a^{\prime \prime \prime}(x)\right\|^{2}|h|^{2}+3 \frac{1}{4}|h|^{4} \\
\leq & 2 \rho(x)^{4}+\rho(x)^{2}|h|^{2}+\frac{1}{12}|h|^{4}+3 \rho(x)^{4}+32^{4} \rho(x)^{2}|h|^{2}+\frac{3}{4}|h|^{4} \\
\leq & 5 \rho(x)^{4}+|h|^{2} \rho(x)^{2}\left(1+32^{4}\right)+|h|^{4}\left(\frac{1}{12}+32^{-2}\right) \\
\leq & 3^{4}(\rho(x)+|h|)^{4} .
\end{aligned}
$$

This implies that

$$
\rho(x+h) \leq 3(\rho(x)+|h|) .
$$

As a consequence, we have for $\|T\| \leq 1, r \geq 0, \rho(x+r \rho(x) T) \leq 3(1+r) \rho(x)$, and thus

$$
|y-x| \leq r \rho(x) \Longrightarrow \rho(y) \leq 3(1+r) \rho(x) .
$$

Moreover if $y=x+r \rho(x) T$ with $r \geq 0$ and $|T| \leq 1$, (A.1.4) gives

$$
\rho(x)=\rho(y-r \rho(x) T) \leq 3(\rho(y)+r \rho(x))
$$

and if $r \leq 1 / 6$ we find $\frac{1}{2} \rho(x) \leq 3 \rho(y) \leq\left(9+\frac{3}{2}\right) \rho(x)$ providing the result of the lemma with $C_{0}=1 / r_{0}=6$.

Remark. When the normalisation condition $\left\|a^{(4)}\right\|_{L^{\infty}} \leq 1$ is not satisfied, it is of course possible to divide $a$ by a constant to get back to that normalization condition. When $\left\|a^{(4)}\right\|_{L^{\infty}} \neq 0$, Lemma A.1.1 is providing the inequalities

$$
\begin{aligned}
& \left\|a^{\prime}(x)\right\|^{4 / 3} \leq 3^{4 / 3}\left(a(x)\left\|a^{(4)}\right\|_{\infty}^{1 / 3}+\left\|a^{\prime \prime}(x)\right\|^{2}\left\|a^{(4)}\right\|_{\infty}^{-2 / 3}\right), \\
& \left\|a^{(3)}(x)\right\|^{4} \leq 4^{4}\left(a(x)\left\|a^{(4)}\right\|_{\infty}^{3}+\left\|a^{\prime \prime}(x)\right\|^{2}\left\|a^{(4)}\right\|_{\infty}^{2}\right) .
\end{aligned}
$$

Note that if $\left\|a^{(4)}\right\|_{L^{\infty}}=0$, i.e. $a^{(4)} \equiv 0, a$ is a polynomial of degree $\leq 3$, and the nonnegativity implies $a^{(3)} \equiv 0$ so that, if its minimum is realized at $0, a$ is the sum of a nonnegative quadratic form and of a nonnegative constant. 
Lemma A.1.3. Let $a, \rho, \Omega$ be as above. Let $\theta$ such that $0<\theta \leq 1 / 2$. If $y \in \Omega$ verifies $a(y) \geq \theta \rho(y)^{4}$, then

$$
|x-y| \leq \theta \rho(y) 2^{-3} \Longrightarrow a(x) \geq \theta \rho(y)^{4} / 2 .
$$

Proof. We note that for $|x-y| \leq r \rho(y)$, using Lemma A.1.1 and Taylor's formula, we have

$$
a(x) \geq a(y)-r \rho(y) 3 \rho(y)^{3}-\frac{1}{2} r^{2} \rho(y)^{2} \rho(y)^{2}-\frac{1}{6} r^{3} \rho(y)^{3} 4 \rho(y)-\frac{1}{24} r^{4} \rho(y)^{4},
$$

implying, for $a(y) \geq \theta \rho(y)^{4}$, that $a(x) \geq \rho(y)^{4}\left(\theta-3 r-\frac{r^{2}}{2}-\frac{2 r^{3}}{3}-\frac{r^{4}}{24}\right)$ and since for $r \leq 1 / 2$, we have $3 r+\frac{r^{2}}{2}+\frac{2 r^{3}}{3}+\frac{r^{4}}{24} \leq r(3+1 / 8+1 / 12+1 / 384) \leq 4 r$ we obtain indeed $a(x) \geq \frac{1}{2} \theta \rho(y)^{4}$ if $r \leq \theta / 8$.

Lemma A.1.4. Let $a, \rho, \Omega$ be as above. There exists $R_{0}>0$ such that if $y \in \Omega$ verifies $a(y)<\rho(y)^{4} / 2$, then there exists a unit vector $T$ such that,

$$
|x-y| \leq R_{0} \rho(y) \Longrightarrow a^{\prime \prime}(x) T^{2} \geq 2^{-1} \rho(y)^{2} .
$$

One can take $R_{0}=10^{-2}$.

Proof. We have $\left\|a^{\prime \prime}(y)\right\| \geq 2^{-1 / 2} \rho(y)^{2}$, so with Remark 3.1.2, we find a unit vector $T$ such that $\left|a^{\prime \prime}(y) T^{2}\right| \geq 2^{-1 / 2} \rho(y)^{2}$. Then we have for all real $s$

$$
\begin{aligned}
0 \leq & a(y+s \rho(y) T) \leq a(y)+s \rho(y) a^{\prime}(y) T+\frac{s^{2}}{2} \rho(y)^{2} a^{\prime \prime}(y) T^{2} \\
& +\frac{s^{3}}{6} \rho(y)^{3} a^{\prime \prime \prime}(y) T^{3}+\frac{s^{4}}{24} \rho(y)^{4} .
\end{aligned}
$$

The quantity $s \rho(y) a^{\prime}(y) T+\frac{s^{3}}{6} \rho(y)^{3} a^{\prime \prime \prime}(y) T^{3}$ is odd in the variable $s$ so that

$$
a(y)+\frac{s^{2}}{2} \rho(y)^{2} a^{\prime \prime}(y) T^{2}+\frac{s^{4}}{24} \rho(y)^{4} \geq\left|s \rho(y) a^{\prime}(y) T+\frac{s^{3}}{6} \rho(y)^{3} a^{\prime \prime \prime}(y) T^{3}\right| \geq 0,
$$

and in particular,

$$
\begin{aligned}
\forall s \neq 0, a^{\prime \prime}(y) T^{2} \geq & -\frac{s^{2}}{12} \rho(y)^{2}-s^{-2} 2 a(y) \rho(y)^{-2} \\
& \Longrightarrow a^{\prime \prime}(y) T^{2} \geq-2 \times 6^{-1 / 2} a(y)^{1 / 2}
\end{aligned}
$$

Since $\left|a^{\prime \prime}(y) T^{2}\right| \geq 2^{-1 / 2} \rho(y)^{2}$, this implies

$$
a^{\prime \prime}(y) T^{2} \geq 2^{-1 / 2} \rho(y)^{2},
$$


otherwise we would have $-2 \times 6^{-1 / 2} a(y)^{1 / 2} \leq a^{\prime \prime}(y) T^{2} \leq-2^{-1 / 2} \rho(y)^{2}$ and thus

$$
a(y)^{1 / 2} \geq 6^{1 / 2} 2^{-3 / 2} \rho(y)^{2} \Longrightarrow a(y) \geq \frac{3}{4} \rho(y)^{4}
$$

which is incompatible with $a(y)<\rho(y)^{4} / 2$. Using the Taylor expansion for $x \mapsto a^{\prime \prime}(x) T^{2}$ yields the following; we write, for $|x-y| \leq \rho(y) s$,

$$
\begin{aligned}
a^{\prime \prime}(x) T^{2} & \geq a^{\prime \prime}(y) T^{2}-|s| \rho(y) 4 \rho(y)-\frac{s^{2}}{2} \rho(y)^{2} \\
& \geq \rho(y)^{2}\left(\frac{1}{\sqrt{2}}-12|s|-3 s^{2} / 2\right) \geq \rho(y)^{2} / 2,
\end{aligned}
$$

provided $|s| \leq 10^{-2}$.

Lemma A.1.5. Let $a, \rho, \Omega, C_{0}, r_{0}, R_{0}$ be as above. There exists a positive constant $\theta_{0}$ such that if $0<\theta \leq \theta_{0}$ and $y \in \Omega$ is such that $a(y)<\theta \rho(y)^{4}$, the following property is true. For all $x$ such that $|x-y| \leq \theta^{1 / 2} \rho(y)$, the function $\tau \mapsto a^{\prime}(x+\tau \rho(y) T) T$ has a unique zero on the interval $\left[-\theta^{1 / 4}, \theta^{1 / 4}\right]$. The constant $\theta_{0}$ is a universal constant that will be chosen also $\leq \min \left(1 / 2, r_{0}^{2}, R_{0}^{4}\right)$.

Proof. From the previous lemma, we know that for $y \in \Omega$ such that $a(y)<\rho(y)^{4} / 2$ then there exists a unit vector $T$ such that,

$$
|x-y| \leq R_{0} \rho(y) \Longrightarrow a^{\prime \prime}(x) T^{2} \geq 2^{-1} \rho(y)^{2} .
$$

The second-order Taylor's formula gives, for $|t| \leq r_{0}$, using (A.1.3),

$$
0 \leq a(y+t \rho(y) T) \leq a(y)+t \rho(y) a^{\prime}(y) T+\frac{\rho(y)^{2} t^{2}}{2} C_{0}^{2} \rho(y)^{2}
$$

and thus

$$
|t| \rho(y)\left|a^{\prime}(y) T\right| \leq a(y)+C_{0}^{2} \rho(y)^{4} t^{2} / 2 \leq \theta \rho(y)^{4}+C_{0}^{2} \rho(y)^{4} t^{2} / 2 .
$$

As a result choosing $t=\theta^{1 / 2}$ (which is indeed smaller than $r_{0}$ ), we get

$$
\left|a^{\prime}(y) T\right| \leq \rho(y)^{3}\left(\theta^{1 / 2}+C_{0}^{2} \theta^{1 / 2} / 2\right) .
$$

We have for $s$ real

$$
\begin{aligned}
a^{\prime}(y+s \rho(y) T) T= & a^{\prime}(y) T+s \rho(y) a^{\prime \prime}(y) T^{2}+\frac{s^{2}}{2} \rho(y)^{2} a^{\prime \prime \prime}(y) T^{3} \\
& +\int_{0}^{1} \frac{1}{2}(1-t)^{2} a^{(4)}(y+t s \rho(y) T) T^{4} d t s^{3} \rho(y)^{3},
\end{aligned}
$$


so that, using (A.1.8), we have

$$
\begin{aligned}
a^{\prime}(y+s \rho(y) T) T & \leq \rho(y)^{3} \theta^{1 / 2} \overbrace{\left(1+C_{0}^{2} / 2\right)}^{=C_{1}}+s \rho(y) a^{\prime \prime}(y) T^{2}+\frac{s^{2}}{2} \rho(y)^{3} 4+\frac{1}{6}|s|^{3} \rho(y)^{3} \\
& \leq \rho(y)^{3}\left(\theta^{1 / 2} C_{1}+s \frac{a^{\prime \prime}(y) T^{2}}{\rho(y)^{2}}+2 s^{2}+\frac{|s|^{3}}{6}\right) .
\end{aligned}
$$

The coefficient of $s$ inside the bracket above belongs to the interval $\left[2^{-1 / 2}, 1\right]$. For $s=-\theta^{1 / 4}$, we get that

$$
a^{\prime}\left(y-\theta^{1 / 4} \rho(y) T\right) T \leq \rho(y)^{3}\left(\theta^{1 / 2} C_{1}-\theta^{1 / 4} 2^{-1 / 2}+2 \theta^{1 / 2}+\frac{|\theta|^{3 / 4}}{6}\right)<0
$$

if $\theta$ is small enough with respect to a universal constant. Since we have also the inequality

$$
\begin{aligned}
a^{\prime}(y+s \rho(y) T) T & \geq-\rho(y)^{3} \theta^{1 / 2} C_{1}+s \rho(y) a^{\prime \prime}(y) T^{2}-\frac{s^{2}}{2} \rho(y)^{3} 4-\frac{1}{6}|s|^{3} \rho(y)^{3} \\
& \geq \rho(y)^{3}\left(-\theta^{1 / 2} C_{1}+s \frac{a^{\prime \prime}(y) T^{2}}{\rho(y)^{2}}-2 s^{2}-\frac{|s|^{3}}{6}\right)
\end{aligned}
$$

the choice $s=\theta^{1 / 4}$ shows that $a^{\prime}\left(y+\theta^{1 / 4} \rho(y) T\right) T>0$. As a result the function $\phi$ defined by $\phi(\tau)=a^{\prime}(y+\tau \rho(y) T) T$ vanishes for some $\tau$ with $|\tau| \leq \theta^{1 / 4} \leq R_{0}$. Moreover, from Lemma A.1.4, its derivative $\phi^{\prime}$ satisfies

$$
\phi^{\prime}(\tau)=a^{\prime \prime}(y+\tau \rho(y) T) T^{2} \rho(y) \geq 2^{-1} \rho(y)^{3}>0,
$$

so that $\phi$ is monotone increasing of $\tau$ on the interval $\left[-\theta^{1 / 4}, \theta^{1 / 4}\right]$, with a unique zero on that interval. Considering now for $|y-x| \leq \theta^{1 / 2} \rho(y)$ the function

$$
\psi(\tau, x)=a^{\prime}(x+\tau \rho(y) T) T
$$

we get that

$$
\phi(\tau)-\theta^{1 / 2} \rho(y) C_{0}^{2} \rho(y)^{2} \leq \psi(\tau, x) \leq \phi(\tau)+\theta^{1 / 2} \rho(y) C_{0}^{2} \rho(y)^{2}
$$

so that the same reasoning as before, we find that for all $x$ such that $|x-y| \leq$ $\theta \rho(y)$, the function $\tau \mapsto a^{\prime}(x+\tau \rho(y) T) T$ has a unique zero on the interval $\left[-\theta^{1 / 4}, \theta^{1 / 4}\right]$, provided that $\theta$ is smaller than a positive universal constant.

Remark A.1.6. Let $a, \rho, \Omega, r_{0}, C_{0}, R_{0}, \theta_{0}$ be as in Lemma A.1.5 and $0<$ $\theta \leq \theta_{0}$. Let $y$ be a point in $\Omega$ such that $a(y)<\theta \rho(y)^{4}$. We may choose the linear orthonormal coordinates such that the vector $T$ given by Lemma A.1.5 
is the first vector of the canonical basis of $\mathbb{R}^{m}$. Then a consequence of Lemma A.1.5 is that, for all $x^{\prime} \in B_{\mathbb{R}^{m-1}}\left(y^{\prime}, \theta^{1 / 2} \rho(y)\right)$ the map $\tau \mapsto \partial_{1} a\left(\tau, x^{\prime}\right)$ has a unique zero $\alpha\left(x^{\prime}\right)$ on the interval $\left[-\theta^{1 / 4} \rho(y)+y_{1}, \theta^{1 / 4} \rho(y)+y_{1}\right]$. We have thus

$$
\left|x^{\prime}-y^{\prime}\right| \leq \theta^{1 / 2} \rho(y) \Longrightarrow \partial_{1} a\left(\alpha\left(x^{\prime}\right), x^{\prime}\right) \equiv 0, \quad\left|\alpha\left(x^{\prime}\right)-y_{1}\right| \leq \theta^{1 / 4} \rho(y) .
$$

From Lemma A.1.4, we get also

$$
\partial_{1}^{2} a\left(\alpha\left(x^{\prime}\right), x^{\prime}\right) \geq \rho(y)^{2} / 2 .
$$

Since the function $\partial_{1} a$ is $C^{2,1}$, the implicit function theorem entails that the function $\alpha$ is $C^{2}$; let us show in fact that $\alpha$ is $C^{2,1}$. Denoting by $\partial_{2}$ the $x^{\prime}$ derivative, with $a$ and its derivatives always evaluated at $x_{1}=\alpha\left(x^{\prime}\right)$, we obtain by differentiating the identity $\partial_{1} a\left(\alpha\left(x^{\prime}\right), x^{\prime}\right) \equiv 0$,

$$
\begin{aligned}
& \alpha^{\prime} \partial_{1}^{2} a+\partial_{1} \partial_{2} a=0, \\
& \alpha^{\prime \prime} \partial_{1}^{2} a+\alpha^{\prime 2} \partial_{1}^{3} a+2 \alpha^{\prime} \partial_{1}^{2} \partial_{2} a+\partial_{1} \partial_{2}^{2} a=0 .
\end{aligned}
$$

The identities (A.1.11-12) give for $\left|x^{\prime}-y^{\prime}\right| \leq \theta^{1 / 2} \rho(y)$, using (A.1.10),

$$
\left\{\begin{array}{l}
\left|\alpha\left(x^{\prime}\right)-y_{1}\right| \leq \theta^{1 / 4} \rho(y) \\
\left|\alpha^{\prime}\left(x^{\prime}\right)\right| \leq 2 \rho(y)^{-2} \rho\left(\alpha\left(x^{\prime}\right), x^{\prime}\right)^{2} \leq 2 C_{0}^{2} \lesssim 1 \\
\left|\alpha^{\prime \prime}\left(x^{\prime}\right)\right| \leq 2 \rho(y)^{-2}\left(4^{2} C_{0}^{4}+4^{2} C_{0}^{2}+12\right) \rho\left(\alpha\left(x^{\prime}\right), x^{\prime}\right) \lesssim \rho(y)^{-1}
\end{array}\right.
$$

We have also the identity, using (A.1.12),

$$
\begin{aligned}
\alpha^{\prime \prime}\left(x^{\prime}\right)= & -\left(\partial_{1}^{2} a\left(\alpha\left(x^{\prime}\right), x^{\prime}\right)\right)^{-1}\left(\alpha^{\prime 2} \partial_{1}^{3} a\left(\alpha\left(x^{\prime}\right), x^{\prime}\right)\right. \\
& \left.+2 \alpha^{\prime} \partial_{1}^{2} \partial_{2} a\left(\alpha\left(x^{\prime}\right), x^{\prime}\right)+\partial_{1} \partial_{2}^{2} a\left(\alpha\left(x^{\prime}\right), x^{\prime}\right)\right),
\end{aligned}
$$

so that the function $\alpha^{\prime \prime}$ is Lipschitz continuous. Applying formally the chain rule from (A.1.12) would give the identity

$\alpha^{\prime \prime \prime} \partial_{1}^{2} a+3 \alpha^{\prime \prime} \alpha^{\prime} \partial_{1}^{3} a+3 \alpha^{\prime \prime} \partial_{1}^{2} \partial_{2} a+\alpha^{\prime 3} \partial_{1}^{4} a+3 \alpha^{\prime 2} \partial_{1}^{3} \partial_{2} a+3 \alpha^{\prime} \partial_{1}^{2} \partial_{2}^{2} a+\partial_{1} \partial_{2}^{3} a=0$

However the meaning of the last four terms above is not clear since the fourth derivative of $a$ is only $L^{\infty}$, so to restrict it to the hypersurface $x_{1}=\alpha\left(x^{\prime}\right)$ does not make sense. In fact, we do not need that, but only the fact that the composition of Lipschitz continuous function gives a Lipschitz continuous functions with the obvious bound on the Lipschitz constant. We start over from (A.1.14) and we write the duality products with a smooth compactly supported 
test function $\chi, a_{\epsilon}$ a regularized $a$,

$$
\begin{aligned}
\left\langle\alpha^{\prime \prime \prime}, \chi\right\rangle & =-\int \alpha^{\prime \prime} \chi^{\prime} d m=\int \chi^{\prime}\left(\alpha^{\prime 2} \partial_{1}^{3} a+2 \alpha^{\prime} \partial_{1}^{2} \partial_{2} a+\partial_{1} \partial_{2}^{2} a\right)\left(\partial_{1}^{2} a\right)^{-2} d m \\
& =\lim _{\epsilon \rightarrow 0} \int \chi^{\prime}\left(\alpha^{\prime 2} \partial_{1}^{3} a_{\epsilon}+2 \alpha^{\prime} \partial_{1}^{2} \partial_{2} a_{\epsilon}+\partial_{1} \partial_{2}^{2} a_{\epsilon}\right)\left(\partial_{1}^{2} a\right)^{-2} d m \\
& =-\lim _{\epsilon \rightarrow 0} \int \chi\left(\left(\alpha^{\prime 2} \partial_{1}^{3} a_{\epsilon}+2 \alpha^{\prime} \partial_{1}^{2} \partial_{2} a_{\epsilon}+\partial_{1} \partial_{2}^{2} a_{\epsilon}\right)\left(\partial_{1}^{2} a\right)^{-2}\right)^{\prime} d m .
\end{aligned}
$$

The computation of the derivative between the parenthesis above, with uniform bounds wih respect to $\epsilon$ gives indeed

$$
\left|\alpha^{\prime \prime \prime}\left(x^{\prime}\right)\right| \lesssim \rho(y)^{-2} .
$$

\section{A.2. More properties of the algebra $\mathcal{A}$}

Lemma A.2.1. Let $b$ be a function in $\mathcal{A}$ and $T \in \mathbb{R}^{2 n}, t \in \mathbb{R}$. Then the functions $\tau_{T} b, b_{t}$ defined by $\tau_{T} b(X)=b(X-T), b_{t}(X)=b(t X)$ belong to $\mathcal{A}$ and

$$
\sup _{T \in \mathbb{R}^{2 n}}\left\|\tau_{T} b\right\|_{\mathcal{A}} \leq C\|b\|_{\mathcal{A}}, \quad\left\|b_{t}\right\|_{\mathcal{A}} \leq(1+|t|)^{2 n} C\|b\|_{\mathcal{A}},
$$

where $C$ depends only on the dimension.

Proof. We check, using that $T=S+j_{0}, j_{0} \in \mathbb{Z}^{2 n}, S \in[0,1]^{2 n}$,

$$
\begin{aligned}
\mathcal{F}\left(\chi_{j} \tau_{T} b\right)(\Xi) & =\int e^{-2 i \pi X \Xi} \chi_{j}(X) b(X-T) d X \\
& =e^{-2 i \pi T \Xi} \int e^{-2 i \pi X \Xi} \chi_{j-j_{0}}(X+S) b(X) d X \\
& =e^{-2 i \pi T \Xi} \int e^{-2 i \pi X \Xi} \chi_{j-j_{0}}(X+S)\left(\sum_{|k| \leq R_{0}} \chi_{j-j_{0}+k}(X) b(X)\right) d X \\
& =e^{-2 i \pi T \Xi} \sum_{|k| \leq R_{0}} \mathcal{F}\left(\left(\tau_{-S} \chi_{j-j_{0}}\right)\left(\chi_{j-j_{0}+k} b\right)\right)(\Xi) \\
& =e^{-2 i \pi T \Xi} \sum_{|k| \leq R_{0}}\left(\mathcal{F}\left(\tau_{-S} \chi_{j-j_{0}}\right) * \mathcal{F}\left(\chi_{j-j_{0}+k} b\right)\right)(\Xi)
\end{aligned}
$$

so that

$$
\begin{aligned}
\left|\mathcal{F}\left(\chi_{j} \tau_{T} b\right)(\Xi)\right| & \leq C_{0} \int\left|\mathcal{F}\left(\tau_{-S} \chi_{j-j_{0}}\right)\left(\Xi-\Xi^{\prime}\right)\right| \omega_{b}\left(\Xi^{\prime}\right) d \Xi^{\prime} \\
& =C_{0} \int\left|\mathcal{F}\left(\chi_{0}\right)\left(\Xi-\Xi^{\prime}\right)\right| \omega_{b}\left(\Xi^{\prime}\right) d \Xi^{\prime}
\end{aligned}
$$


entailing $\int \sup _{j \in \mathbb{Z}^{2 n}}\left|\mathcal{F}\left(\chi_{j} \tau_{T} b\right)(\Xi)\right| d \Xi \leq C_{0}\left\|\widehat{\chi_{0}}\right\|_{L^{1}}\|b\|_{\mathcal{A}}$ and the first part of (A.2.1). The second part is obvious if $t=0$ since $\mathcal{A}$ is continuously embedded in $C^{0} \cap L^{\infty}$ (Proposition 1.2.1). Assuming $t \neq 0$, we look now at

$$
\begin{aligned}
\mathcal{F}\left(\chi_{j} b_{t}\right)(\Xi) & =\int e^{-2 i \pi X \Xi} \chi_{0}(X-j) b(t X) d X \\
& =\sum_{k \in \mathbb{Z}^{2 n}} \int e^{-2 i \pi X \Xi} \chi_{0}(X-j) \chi_{k}(t X) b(t X) d X
\end{aligned}
$$

and since on the support of the integrand, we have $|X-j| \leq R_{0}, \quad|k-t X| \leq R_{0}$ and thus $|k-t j| \leq R_{0}+|t| R_{0}$, we get

$$
\begin{aligned}
& \mathcal{F}\left(\chi_{j} b_{t}\right)(\Xi)=\int e^{-2 i \pi X \Xi} \chi_{0}(X-j) b(t X) d X \\
& =\sum_{\substack{k \\
|k-t j| \leq R_{0}(1+|t|)}} \int e^{-2 i \pi X \Xi} \chi_{0}(X-j) b(t X) \chi_{k}(t X) d X \\
& =\sum_{|k-t j| \leq R_{0}(1+|t|)} \iint e^{-2 i \pi t^{-1} X \Xi} \widehat{\chi_{0}}(N) e^{2 i \pi N\left(t^{-1} X-j\right)} \\
& \times\left(\chi_{k} b\right)(X) d X d N|t|^{-2 n}
\end{aligned}
$$

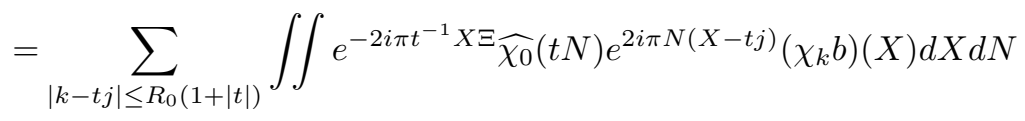

$$
\begin{aligned}
& =\sum_{|k-t j| \leq R_{0}(1+|t|)} \iint \widehat{\chi_{0}}(t N) e^{-2 i \pi N t j} e^{2 i \pi X\left(N-t^{-1} \Xi\right)}\left(\chi_{k} b\right)(X) d X d N \\
& =\sum_{|k-t j| \leq R_{0}(1+|t|)} \iint \widehat{\chi_{0}}(t N+\Xi) e^{-2 i \pi N t j} e^{-2 i \pi \Xi j} e^{2 i \pi X N} \\
& \times\left(\chi_{k} b\right)(X) d X d N \\
& =\sum_{|k-t j| \leq R_{0}(1+|t|)} \int \widehat{\chi_{0}}(-t N+\Xi) e^{2 i \pi N t j} e^{-2 i \pi \Xi j} \\
& \times\left(\int e^{-2 i \pi X N}\left(\chi_{k} b\right)(X) d X\right) d N
\end{aligned}
$$

so that

$$
\begin{aligned}
\left|\mathcal{F}\left(\chi_{j} b_{t}\right)(\Xi)\right| & \leq \sum_{|k-t j| \leq R_{0}(1+|t|)} \int\left|\widehat{\chi_{0}}(-t N+\Xi)\right|\left|\int e^{-2 i \pi X N}\left(\chi_{k} b\right)(X) d X\right| d N \\
& \leq C_{n} R_{0}^{2 n} \int\left|\widehat{\chi_{0}}(-t N+\Xi)\right| \omega_{b}(N) d N(1+|t|)^{2 n},
\end{aligned}
$$


and finally the sought result $\int \sup _{j}\left|\mathcal{F}\left(\chi_{j} b_{t}\right)(\Xi)\right| d \Xi \leq C_{n} R_{0}^{2 n}(1+|t|)^{2 n}\left\|\widehat{\chi_{0}}\right\|_{L^{1}}$ $\times\|b\|_{\mathcal{A}}$.

\section{A.3. On Leibniz formulæ}

Let $a$ be a function in $L_{\text {loc }}^{1}$ of some open set $\Omega$ of $\mathbb{R}^{m}$ and let $u$ be a locally Lipschitz continuous function on $\Omega$. Although $a^{\prime}$ may be a distribution of order 1 and $u$ is not $C^{1}$, it is possible to define the product $T=a^{\prime} u$ as follows ( $\varphi$ is a test function):

$$
\langle T, \varphi\rangle=-\int a\left(u^{\prime} \varphi+u \varphi^{\prime}\right) d x
$$

so that $T$ is a distribution of order 1 satisfying the identity $(a u)^{\prime}=T+a u^{\prime}$. As a matter of fact, we have $\left\langle(a u)^{\prime}, \varphi\right\rangle=-\int a u \varphi^{\prime} d x=\langle T, \varphi\rangle+\int a u^{\prime} \varphi d x=\langle T+$ $\left.a u^{\prime}, \varphi\right\rangle$. It means in particular that one can multiply the first-order distribution $\frac{d}{d x}(\ln |x|)=\operatorname{pv} \frac{1}{x}$ by the Lipschitz continuous function $|x|$ and get $\left(\mathrm{pv} \frac{1}{x}\right)|x|=$ $\frac{d}{d x}((\ln |x|)|x|)-(\ln |x|) \operatorname{sign} x=\operatorname{sign} x$ as it is easily verified. On the other hand it is not possible to multiply the first order distribution $\delta_{0}^{\prime}$ by the Lipschitz continuous function $|x|$.

\section{A.4. Symmetric $k$-tensors as sum of $k$-th powers}

Since the symmetrized products of $T_{1} \otimes \cdots \otimes T_{k}$ can be written as a linear combination of $k$-th powers, the norm of the $k$-linear symmetric form $A$ given by $\|A\|=\sup _{\|T\|=1}\left|A T^{k}\right|$ is equivalent to the natural norm

$$
\|A\|=\sup _{\substack{\left\|T_{j}\right\|=1, 1 \leq j \leq k}}\left|A T_{1} \ldots T_{k}\right|
$$

and we have the inequalities $\|A\| \leq\|A\| \leq \kappa_{k}\|A\|$ with a constant $\kappa_{k}$ depending only on $k$. The best constant constant in general is $\kappa_{k}=k^{k} / k !$. In fact, in a commutative algebra on a field with characteristic 0 , using the polarization formula, the products $T_{1} \ldots T_{k}$ are linear combination of $k$-th powers

$$
T_{1} T_{2} \ldots T_{k}=\frac{1}{2^{k} k !} \sum_{\epsilon_{j}= \pm 1} \epsilon_{1} \ldots \epsilon_{k}\left(\epsilon_{1} T_{1}+\cdots+\epsilon_{k} T_{k}\right)^{k} .
$$

Using the triangle inequality, we get $\|A\| \leq \frac{1}{2^{k} k !} 2^{k} k^{k}\|A\|$, and thus $\kappa_{k} \leq \frac{k^{k}}{k !}$. On the other hand, for $T_{j} \in \mathbb{R}^{k}$ and $A$ defined by

$$
A\left(T_{1}, \ldots, T_{k}\right)=\frac{1}{k !} \sum_{\sigma \in \mathcal{S}_{k}} T_{\sigma(1), 1} \ldots T_{\sigma(k), k},
$$


we have $A\left(e_{1}, \ldots, e_{k}\right)=1 / k$ ! so that $\|A\| \geq \frac{1}{k !}$ and for $\theta \in \mathbb{R}^{k}$ (with the norm $\left.\sum\left|\theta_{j}\right|\right)$

$$
\left|A \theta^{k}\right|=\left|\theta_{1} \ldots \theta_{k}\right| \leq\left(\frac{\sum\left|\theta_{j}\right|}{k}\right)^{k} \Longrightarrow\|A\| \leq k^{-k},
$$

so that $\kappa_{k} \geq \frac{\|A\|}{\|A\|} \geq \frac{k^{k}}{k !}$.

\section{A.5. From discrete sums to finite sums}

At the end of the proof of Theorem 3.1.1, we have established that

$$
a(x)=\sum_{1 \leq j \leq 1+N_{m-1}} \sum_{\nu \in \mathbb{N}} b_{\nu, j}(x)^{2} \varphi_{\nu}(x)^{2}
$$

with $\left(\varphi_{\nu}\right)$ satisfying the properties of Lemma 3.1 .3 and the $b_{\nu, j}$ are $C^{1,1}$ functions such that

$$
\left|b_{\nu, j}^{(l)}\right| \leq c_{0} \rho_{\nu}^{2-l}, \quad 0 \leq l \leq 2, \quad\left|\left(b_{\nu, j}^{\prime} b_{\nu, j}^{\prime \prime}\right)^{\prime}\right| \leq c_{0} \quad\left|\left(b_{\nu, j} b_{\nu, j}^{\prime \prime}\right)^{\prime \prime}\right| \leq c_{0},
$$

where $c_{0}$ is a universal constant (we keep the normalization assumption $\left.\left\|a^{(4)}\right\|_{L^{\infty}\left(\mathbb{R}^{m}\right)} \leq 1\right)$. We want to write $a$ as a finite sum with similar properties, using the slow variation of the metric $|d x|^{2} / \rho(x)^{2}$. We are given a positive number $r \leq r_{0}^{\prime}$, where $r_{0}^{\prime}$ is defined in Lemma 3.1.3. We define a sequence $\left(x_{\nu}\right)$ and balls $U_{\nu}$ as in that lemma.

$\cdot \mathcal{N}_{1}=$ maximal subset of $\mathbb{N}$ containing 0 such that for $\nu^{\prime} \neq \nu^{\prime \prime}$ both in $\mathcal{N}_{1}$,

$$
U_{\nu^{\prime}} \cap U_{\nu^{\prime \prime}}=\emptyset . \quad \text { Let } \nu_{2}=\min \mathcal{N}_{1}^{c} .
$$

- $\mathcal{N}_{2}=$ maximal subset of $\mathcal{N}_{1}^{c}$ containing $\nu_{2}$ such that for $\nu^{\prime} \neq \nu^{\prime \prime}$ both in $\mathcal{N}_{2}$,

$$
\begin{aligned}
& U_{\nu^{\prime}} \cap U_{\nu^{\prime \prime}}=\emptyset . \quad \text { Let } \nu_{3}=\min \left(\mathcal{N}_{1} \cup \mathcal{N}_{2}\right)^{c} . \\
& \ldots \quad \text { Let } \nu_{k+1}=\min \left(\mathcal{N}_{1} \cup \cdots \cup \mathcal{N}_{k}\right)^{c} .
\end{aligned}
$$

$\cdot \mathcal{N}_{k+1}=$ maximal subset of $\left(\mathcal{N}_{1} \cup \cdots \cup \mathcal{N}_{k}\right)^{c}$ containing $\nu_{k+1}$

such that for $\nu^{\prime} \neq \nu^{\prime \prime}$ both in $\mathcal{N}_{k+1}$,

$$
U_{\nu^{\prime}} \cap U_{\nu^{\prime \prime}}=\emptyset \text {. Let } \nu_{k+2}=\min \left(\mathcal{N}_{1} \cup \cdots \cup \mathcal{N}_{k+1}\right)^{c} \text {. }
$$

We observe the following.

- The sets $\mathcal{N}_{j}$ are two by two disjoint.

- For all $j, k$ such that $1 \leq j \leq k$, there exists $\nu \in \mathcal{N}_{j}$ so that $U_{\nu} \cap U_{\nu_{k+1}} \neq$ $\emptyset$ : otherwise, we could find $1 \leq j \leq k$ so that for all $\nu \in \mathcal{N}_{j}, U_{\nu} \cap U_{\nu_{k+1}}=\emptyset$, 
so that the set $\mathcal{N}_{j} \cup\left\{\nu_{k+1}\right\}$ would satisfy the property that the maximal $\mathcal{N}_{j}$ should satisfy.

- For $k$ large enough, we have $\mathcal{N}_{1} \cup \cdots \cup \mathcal{N}_{k}=\mathbb{N}$ : otherwise $\nu_{k+1}$ is always well-defined and using the property above, we get that one can find $\mu_{j} \in \mathcal{N}_{j}, 1 \leq j \leq k$, so that $U_{\mu_{j}} \cap U_{\nu_{k+1}} \neq \emptyset$. As a consequence, for $1 \leq j \leq k$, we find $y_{j} \in U_{\mu_{j}}$ such that

$$
\begin{aligned}
& \left|x_{\mu_{j}}-y_{j}\right| \leq r \rho\left(x_{\mu_{j}}\right) \leq C_{0} r \rho\left(y_{j}\right), \\
& \left|x_{\nu_{k+1}}-y_{j}\right| \leq r \rho\left(x_{\nu_{k+1}}\right) \leq C_{0} r \rho\left(y_{j}\right) \leq C_{0}^{2} r \rho\left(x_{\nu_{k+1}}\right)
\end{aligned}
$$

and thus

$$
\left|x_{\nu_{k+1}}-x_{\mu_{j}}\right| \leq\left(C_{0}^{2} r+r\right) \rho\left(x_{\nu_{k+1}}\right),
$$

with distinct $\mu_{j}$ (they belong to two by two disjoint sets). On the other hand, we know by construction (see Lemma 1.4.9 in [H2]) that there exists a positive $r_{1}$ such that, for $\nu^{\prime} \neq \nu^{\prime \prime}$,

$$
\left\|x_{\nu^{\prime}}-x_{\nu^{\prime \prime}}\right\| \geq r_{1} \rho\left(x_{\nu^{\prime}}\right)
$$

so that, with a fixed $r_{2}>0$, the balls $\left(B\left(x_{\mu_{j}}, r_{2} \rho\left(x_{\mu_{j}}\right)\right)\right)_{1 \leq j \leq k}$ are two by two disjoint as well as $\left(B\left(x_{\mu_{j}}, r_{3} \rho\left(x_{\nu_{k+1}}\right)\right)\right)_{1 \leq j \leq k}$ with a fixed positive $r_{3}$. Thanks to (A.5.3), they are also all included in $B\left(x_{\nu_{k+1}}, r_{4} \rho\left(x_{\nu_{k+1}}\right)\right)$ with a fixed positive $r_{4}$ so that $k \leq r_{4}^{m} / r_{3}^{m}$ and thus $k$ is bounded. We can thus write, with $M_{m}=\lambda_{0}^{m}$, since the balls $U_{\nu}\left(\supset \operatorname{supp} \varphi_{\nu}\right)$ are two by two disjoint for $\nu$ running in each $\mathcal{N}_{k}$,

$$
a=\sum_{1 \leq j \leq 1+N_{m-1}} \sum_{1 \leq k \leq M_{m}}\left(\sum_{\nu \in \mathcal{N}_{k}} b_{\nu, j} \varphi_{\nu}\right)^{2}
$$

and defining $B_{j, k}=\sum_{\nu \in \mathcal{N}_{k}} b_{\nu, j} \varphi_{\nu}$ we get

$$
a=\sum_{1 \leq j \leq 1+N_{m-1}} \sum_{1 \leq k \leq M_{m}} B_{j, k}^{2}
$$

with $\left|B_{j, k}^{\prime \prime}\right| \leq \sum_{\nu \in \mathcal{N}_{k}} c_{0} \psi_{\nu} \lesssim 1$. Moreover the identities

$$
\begin{aligned}
& \left(B_{j, k}^{\prime} B_{j, k}^{\prime \prime}\right)^{\prime}=\sum_{\nu \in \mathcal{N}_{k}}\left(\left(b_{\nu, j} \varphi_{\nu}\right)^{\prime}\left(b_{\nu, j} \varphi_{\nu}\right)^{\prime \prime}\right)^{\prime} \psi_{\nu}, \\
& \left(B_{j, k} B_{j, k}^{\prime \prime}\right)^{\prime \prime}=\sum_{\nu \in \mathcal{N}_{k}}\left(\left(b_{\nu, j} \varphi_{\nu}\right)\left(b_{\nu, j} \varphi_{\nu}\right)^{\prime \prime}\right)^{\prime \prime} \psi_{\nu}
\end{aligned}
$$


yield the sought estimates on the derivatives. As a final question, one may ask for some estimate of the Pythagorean number, i.e. the number of squares necessary for the decomposition. From the formula (A.5.4), we have the estimate

$$
N_{m} \leq\left(1+N_{m-1}\right) \lambda_{0}^{m}, \quad \lambda_{0} \text { universal constant }
$$

which gives $N_{m} \leq \mu_{0}^{m^{2}}$, which is probably a very crude estimate, compared to the exponential bound known for the Artin theorem of decomposition as sum of squares of nonnegative rational fractions. As a matter of fact, a recent paper of Bony [Bo2] is providing the equality $N_{1}=2$, which is optimal in view of the Glaeser counterexample ([Gl]); however his proof is much more involved than our argument as exposed above with our set of indices $\mathcal{N}_{k}$.

\section{References}

$[\mathrm{AM}]$ H. Ando and Y. Morimoto, Wick calculus and the Cauchy problem for some dispersive equations, Osaka J. Math. 39 (2002), no. 1, 123-147.

[Be] F. A. Berezin, Quantization, Math. USSR, Izvest. 8 (1974), 1109-1165.

[Bo1] J.-M. Bony Sur l'inégalité de Fefferman-Phong in Seminaire: Équations aux Dérivées Partielles, 1998-1999, Exp. III, 16 pp, École Polytech., Palaiseau.

[Bo2] D Décomposition des fonctions positives en sommes de carrés, in Journées "Équations aux Dérivées Partielles", 2004, Exp. III, 8 pp, École Polytech., Palaiseau.

[BL] J.-M. Bony and N. Lerner, Quantification asymptotique et microlocalisations d'ordre supérieur. I, Ann. Sci. École Norm. Sup. (4) 22 (1989), no. 3, 377-433.

[B1] A. Boulkhemair, Remarks on a Wiener type pseudodifferential algebra and Fourier integral operators, Math. Res. Lett. 4 (1997), no. 1, 53-67.

[B2] — $L^{2}$ estimates for Weyl quantization, J. Funct. Anal. 165 (1999), no. 1, 173204.

[CF] A. Córdoba and C. Fefferman, Wave packets and Fourier integral operators, Comm. Partial Differential Equations 3 (1978), no. 11, 979-1005.

[FP] C. Fefferman and D. H. Phong, On positivity of pseudo-differential operators, Proc. Nat. Acad. Sci. U.S.A. 75 (1978), no. 10, 4673-4674.

[Gl] G. Glaeser, Racine carrée d'une fonction différentiable, Ann. Inst. Fourier (Grenoble) 13 (1963), fasc. 2, 203-210.

[Gu] P. Guan, $C^{2}$ a priori estimates for degenerate Monge-Ampère equations, Duke Math. J. 86 (1997), no. 2, 323-346.

[GL] K. Gröchenig and M. Leinert, Wiener's lemma for twisted convolution and Gabor frames, J. Amer. Math. Soc. 17 (2004), no. 1, 1-18 (electronic).

[Hé] F. Hérau, Melin-Hörmander inequality in a Wiener type pseudo-differential algebra, Ark. Mat. 39 (2001), no. 2, 311-338.

[H1] L. Hörmander, The Weyl calculus of pseudodifferential operators, Comm. Pure Appl. Math. 32 (1979), no. 3, 360-444.

[H2] $\longrightarrow$, The analysis of linear partial differential operators I-IV, Springer, Berlin Verlag, 1983-1985.

[Ke] O. D. Kellogg, On bounded polynomials in several variables, Math. Z. 27 (1928), no. 1, 55-64.

[L1] N. Lerner, Energy methods via coherent states and advanced pseudo-differential calculus, in Multidimensional complex analysis and partial differential equations (São 
Carlos, 1995), 177-201, Contemp. Math., 205, Amer. Math. Soc., Providence, RI, 1997.

[L2] N. Lerner, Perturbation and energy estimates, Ann. Sci. École Norm. Sup. (4) 31 (1998), no. 6, 843-886.

[L3] Solving pseudo-differential equations, in Proceedings of the International Congress of Mathematicians, Vol. II (Beijing, 2002), 711-720, Higher Ed. Press, Beijing.

[LN] N. Lerner and J. Nourrigat, Lower bounds for pseudo-differential operators, Ann. Inst. Fourier (Grenoble) 40 (1990), no. 3, 657-682.

[S1] J. Sjöstrand, An algebra of pseudodifferential operators, Math. Res. Lett. 1 (1994), no. 2, 185-192.

[S2] Wiener type algebras of pseudodifferential operators, in Séminaire sur les Équations aux Dérivées Partielles, 1994-1995, Exp. IV, 21 pp, École Polytech., Palaiseau.

[Sh] M. Shubin Pseudo-differential operators and spectral theory, Springer-Verlag, 1985.

[Ta] D. Tataru, On the Fefferman-Phong inequality and related problems, Comm. Partial Differential Equations 27 (2002), no. 11-12, 2101-2138.

[Un] A. Unterberger, Oscillateur harmonique et opérateurs pseudo-différentiels, Ann. Inst. Fourier (Grenoble) 29 (1979), no. 3, xi, 201-221. 